\title{
Periodic homogenization of a Lévy-type process with small jumps
}

\author{
Nikola SANDrić, IVANA VALENTIĆ AND JiAN WANG
}

Abstract. In this article, we consider the problem of periodic homogenization of a Feller process generated by a pseudo-differential operator, the so-called Lévy-type process. Under the assumptions that the generator has rapidly periodically oscillating coefficients, and that it admits "small jumps" only (that is, the jump kernel has finite second moment), we prove that the appropriately centered and scaled process converges weakly to a Brownian motion with covariance matrix given in terms of the coefficients of the generator. The presented results generalize the classical and well-known results related to periodic homogenization of a diffusion process.

\section{Introduction}

The classical reaction-diffusion equation

$$
\partial_{t} p(t, x)=\left\langle b(x), \nabla_{x} p(t, x)\right\rangle+\frac{1}{2} \operatorname{Tr} c(x) \nabla_{x}^{2} p(t, x)+r(p(t, x))
$$

describes the evolution of population density due to random displacement of individuals (diffusion term), movement of individuals within the environment (drift term), and their reproduction (reaction term). In order to characterize long-range effects the diffusion and drift terms are naturally replaced by an integro-differential operator of the following form

$$
\begin{aligned}
\mathcal{L} f(x)= & \langle b(x), \nabla f(x)\rangle+\frac{1}{2} \operatorname{Tr} c(x) \nabla^{2} f(x) \\
& +\int_{\mathbb{R}^{d}}\left(f(x+y)-f(x)-\langle y, \nabla f(x)\rangle \mathbb{1}_{B_{1}(0)}(y)\right) v(x, \mathrm{~d} y),
\end{aligned}
$$

where $v(x, \mathrm{~d} y)$ is a non-negative Borel kernel which describes these effects, that is, it quantifies the property that an individual at $x$ jumps to $x+\mathrm{d} y$.

The main goal of this article is to discuss periodic homogenization of the operator $\mathcal{L}$, with kernel $v(x, \mathrm{~d} y)$ admitting "small jumps" only (that is, having finite second moment). Our approach is based on probabilistic techniques. More precisely, we discuss periodic homogenization of the stochastic (Markov) process $\left\{X_{t}\right\}_{t \geq 0}$ in periodic

Mathematics Subject Classification: 35S15, 47G20, 60F17, 60J75

Keywords: Feller process, Homogenization, Lévy-type process, Pseudo-differential operator, Semimartingale characteristics. 
medium, generated by $\mathcal{L}$. We focus to the case when $\left\{X_{t}\right\}_{t \geq 0}$ is a so-called Lévy-type process or, equivalently, when $\mathcal{L}$ is a pseudo-differential operator (see below for details). Roughly speaking, we show that the appropriately centered and scaled process $\left\{X_{t}\right\}_{t \geq 0}$ :

$$
\left\{\varepsilon X_{\varepsilon^{-2} t}-\varepsilon^{-1} \bar{b}^{*} t\right\}_{t \geq 0},
$$

for some $\overline{b^{*}} \in \mathbb{R}^{d}$, converges, as $\varepsilon \rightarrow 0$, in the path space endowed with the Skorohod $\mathrm{J}_{1}$-topology to a $d$-dimensional zero-drift Brownian motion determined by covariance matrix of the form

$$
\begin{aligned}
\Sigma:=( & \int_{\mathbb{T}_{\tau}^{d}} \sum_{k, l=1}^{d}\left(\delta_{k i}-\partial_{k} \beta_{i}(x)\right) c_{k l}(x)\left(\delta_{l j}-\partial_{l} \beta_{j}(x)\right) \pi(\mathrm{d} x) \\
& +\int_{\mathbb{T}_{\tau}^{d}} \int_{\mathbb{R}^{d}} y_{i} y_{j} v(x, \mathrm{~d} y) \pi(\mathrm{d} x) \\
& +\int_{\mathbb{T}_{\tau}^{d}} \int_{\mathbb{R}^{d}}\left(\beta_{i}(x+y)-\beta_{i}(x)\right)\left(\beta_{j}(x+y)-\beta_{j}(x)\right) v(x, \mathrm{~d} y) \pi(\mathrm{d} x) \\
& \left.-2 \int_{\mathbb{T}_{\tau}^{d}} \int_{\mathbb{R}^{d}} y_{i}\left(\beta_{j}(x+y)-\beta_{j}(x)\right) v(x, \mathrm{~d} y) \pi(\mathrm{d} x)\right)_{1 \leq i, j \leq d},
\end{aligned}
$$

(see Theorem 1.4 for details). Equivalently, according to [14, Theorem 7.1],

$$
\lim _{\varepsilon \rightarrow 0}\left\|\mathcal{L}_{\varepsilon} f-\varepsilon^{-1}\left\langle\overline{b^{*}}, \nabla f\right\rangle-2^{-1} \operatorname{Tr} \Sigma \nabla^{2} f\right\|_{\infty}=0, \quad f \in C_{c}^{\infty}\left(\mathbb{R}^{d}\right),
$$

where

$$
\begin{aligned}
\mathcal{L}_{\varepsilon} f(x)= & \varepsilon^{-1}\langle b(x / \varepsilon), \nabla f(x)\rangle+\frac{1}{2} \operatorname{Tr} c(x / \varepsilon) \nabla^{2} f(x) \\
& +\varepsilon^{-2} \int_{\mathbb{R}^{d}}\left(f(x+\varepsilon y)-f(x)-\varepsilon\langle y, \nabla f(x)\rangle \mathbb{1}_{B_{1}(0)}(y)\right) v(x / \varepsilon, \mathrm{d} y) .
\end{aligned}
$$

Let us remark that when $b(x) \equiv 0$ and $v(x, \mathrm{~d} y)$ is symmetric for all $x \in \mathbb{R}^{d}$, centralization in Eq. (1.2) is not necessary (that is, one can take $\overline{b^{*}}=0$ ), and $\beta(x) \equiv 0$ in Eq. (1.3). Thus, in this case, $\Sigma$ is reduced to

$$
\left(\int_{\mathbb{T}_{\tau}^{d}} c_{i j}(x) \pi(\mathrm{d} x)+\int_{\mathbb{T}_{\tau}^{d}} \int_{\mathbb{R}^{d}} y_{i} y_{j} v(x, \mathrm{~d} y) \pi(\mathrm{d} x)\right)_{1 \leq i, j \leq d},
$$

(see [67] for more details).

Preliminaries on Lévy-Type processes. Let $\left(\Omega, \mathcal{F},\left\{\mathbb{P}_{x}\right\}_{x \in \mathbb{R}^{d}},\left\{\mathcal{F}_{t}\right\}_{t \geq 0},\left\{\theta_{t}\right\}_{t \geq 0}\right.$, $\left.\left\{X_{t}\right\}_{t \geq 0}\right)$, denoted by $\left\{X_{t}\right\}_{t \geq 0}$ in the sequel, be a Markov process on state space $\left(\mathbb{R}^{d}, \mathcal{B}\left(\mathbb{R}^{d}\right)\right.$ ) (see [13]). Here, $d \geq 1$, and $\mathcal{B}\left(\mathbb{R}^{d}\right)$ denotes the Borel $\sigma$-algebra on $\mathbb{R}^{d}$. Due to the Markov property, the associated family of linear operators $\left\{P_{t}\right\}_{t \geq 0}$ on $B_{b}\left(\mathbb{R}^{d}\right)$ (the space of bounded and Borel measurable functions), defined by

$$
P_{t} f(x):=\mathbb{E}_{x}\left[f\left(X_{t}\right)\right], \quad t \geq 0, x \in \mathbb{R}^{d}, f \in B_{b}\left(\mathbb{R}^{d}\right),
$$


forms a semigroup on the Banach space $\left(B_{b}\left(\mathbb{R}^{d}\right),\|\cdot\|_{\infty}\right)$, that is, $P_{0}=\operatorname{Id}$ and $P_{s} \circ P_{t}=$ $P_{s+t}$ for all $s, t \geq 0$. Here, $\mathbb{E}_{x}$ stands for the expectation with respect to $\mathbb{P}_{x}(\mathrm{~d} \omega)$, $x \in \mathbb{R}^{d}$, and $\|\cdot\|_{\infty}$ and Id denote the supremum norm and the identity operator, respectively, on the space $B_{b}\left(\mathbb{R}^{d}\right)$. Moreover, the semigroup $\left\{P_{t}\right\}_{t \geq 0}$ is contractive $\left(\left\|P_{t} f\right\|_{\infty} \leq\|f\|_{\infty}\right.$ for all $t \geq 0$ and $\left.f \in B_{b}\left(\mathbb{R}^{d}\right)\right)$ and positivity preserving $\left(P_{t} f \geq\right.$ 0 for all $t \geq 0$ and $f \in B_{b}\left(\mathbb{R}^{d}\right)$ satisfying $\left.f \geq 0\right)$. The infinitesimal generator $\left(\mathcal{A}^{b}, \mathcal{D}_{\mathcal{A}^{b}}\right)$ of the semigroup $\left\{P_{t}\right\}_{t \geq 0}$ (or of the process $\left\{X_{t}\right\}_{t \geq 0}$ ) is a linear operator $\mathcal{A}^{b}: \mathcal{D}_{\mathcal{A}^{b}} \rightarrow B_{b}\left(\mathbb{R}^{d}\right)$ defined by

$\mathcal{A}^{b} f:=\lim _{t \rightarrow 0} \frac{P_{t} f-f}{t}, \quad f \in \mathcal{D}_{\mathcal{A}^{b}}:=\left\{f \in B_{b}\left(\mathbb{R}^{d}\right): \lim _{t \rightarrow 0} \frac{P_{t} f-f}{t}\right.$ exists in $\left.\|\cdot\|_{\infty}\right\}$.

We call $\left(\mathcal{A}^{b}, \mathcal{D}_{\mathcal{A}^{b}}\right)$ the $B_{b}$-generator for short. A Markov process $\left\{X_{t}\right\}_{t \geq 0}$ is said to be a Feller process if its corresponding semigroup $\left\{P_{t}\right\}_{t \geq 0}$ forms a Feller semigroup. This means that

(i) $\left\{P_{t}\right\}_{t \geq 0}$ enjoys the Feller property, that is, $P_{t}\left(C_{\infty}\left(\mathbb{R}^{d}\right)\right) \subseteq C_{\infty}\left(\mathbb{R}^{d}\right)$ for all $t \geq 0$

(ii) $\left\{P_{t}\right\}_{t \geq 0}$ is strongly continuous, that is, $\lim _{t \rightarrow 0}\left\|P_{t} f-f\right\|_{\infty}=0$ for all $f \in$ $C_{\infty}\left(\mathbb{R}^{d}\right)$.

Here, $C_{\infty}\left(\mathbb{R}^{d}\right)$ denotes the space of continuous functions vanishing at infinity. Recall also that a Markov process $\left\{X_{t}\right\}_{t \geq 0}$ is said to be a $C_{b}$-Feller (resp. strong Feller) process if the corresponding semigroup $\left\{P_{t}\right\}_{t \geq 0}$ satisfies $P_{t} f \in C_{b}\left(\mathbb{R}^{d}\right)$ for all $t>0$ and all $f \in C_{b}\left(\mathbb{R}^{d}\right)$ [resp. $\left.f \in B_{b}\left(\mathbb{R}^{d}\right)\right]$, where $C_{b}\left(\mathbb{R}^{d}\right):=C\left(\mathbb{R}^{d}\right) \cap B_{b}\left(\mathbb{R}^{d}\right)$. Note that every Feller semigroup $\left\{P_{t}\right\}_{t \geq 0}$ can be uniquely extended to $B_{b}\left(\mathbb{R}^{d}\right)$ (see [69, Section 3]). For notational simplicity, we denote this extension by $\left\{P_{t}\right\}_{t \geq 0}$ again. Also, let us remark that every Feller process (admits a modification that) has càdlàg sample paths and possesses the strong Markov property (see [42, Theorems 3.4.19 and 3.5.14]). Further, in the case of Feller processes, we call $\left(\mathcal{A}^{\infty}, \mathcal{D}_{\mathcal{A}^{\infty}}\right):=\left(\mathcal{A}^{b}, \mathcal{D}_{\mathcal{A}^{b}} \cap C_{\infty}\left(\mathbb{R}^{d}\right)\right)$ the Feller generator for short. Observe that in this case $\mathcal{D}_{\mathcal{A}} \subseteq \subseteq C_{\infty}\left(\mathbb{R}^{d}\right)$ and $\mathcal{A}^{\infty}\left(\mathcal{D}_{\mathcal{A}}{ }^{\infty}\right) \subseteq$ $C_{\infty}\left(\mathbb{R}^{d}\right)$. If the set of smooth functions with compact support $C_{c}^{\infty}\left(\mathbb{R}^{d}\right)$ is contained in $\mathcal{D}_{\mathcal{A}}$, that is, if the Feller generator $\left(\mathcal{A}^{\infty}, \mathcal{D}_{\mathcal{A}^{\infty}}\right)$ of the Feller process $\left\{X_{t}\right\}_{t \geq 0}$ satisfies (LTP1) $C_{c}^{\infty}\left(\mathbb{R}^{d}\right) \subseteq \mathcal{D}_{\mathcal{A}}$,

then, according to [24, Theorem 3.4], $\left.\mathcal{A}^{\infty}\right|_{C_{c}^{\infty}\left(\mathbb{R}^{d}\right)}$ is a pseudo-differential operator, that is, it can be written in the form

$$
\left.\mathcal{A}^{\infty}\right|_{C_{c}^{\infty}\left(\mathbb{R}^{d}\right)} f(x)=-\int_{\mathbb{R}^{d}} q(x, \xi) \mathrm{e}^{i\langle\xi, x\rangle} \hat{f}(\xi) \mathrm{d} \xi,
$$

where $\hat{f}(\xi):=(2 \pi)^{-d} \int_{\mathbb{R}^{d}} \mathrm{e}^{-i\langle\xi, x\rangle} f(x) \mathrm{d} x$ denotes the Fourier transform of the function $f(x)$. The function $q: \mathbb{R}^{d} \times \mathbb{R}^{d} \rightarrow \mathbb{C}$ is called the symbol of the pseudodifferential operator. It is measurable and locally bounded in $(x, \xi)$, and is continuous and negative definite as a function of $\xi$. Hence, by [41, Theorem 3.7.7], the function 
$\xi \mapsto q(x, \xi)$ has for each $x \in \mathbb{R}^{d}$ the following Lévy-Khintchine representation

$$
\begin{aligned}
q(x, \xi)= & a(x)-i\langle\xi, b(x)\rangle+\frac{1}{2}\langle\xi, c(x) \xi\rangle \\
& +\int_{\mathbb{R}^{d}}\left(1-\mathrm{e}^{i\langle\xi, y\rangle}+i\langle\xi, y\rangle \mathbb{1}_{B_{1}(0)}(y)\right) v(x, \mathrm{~d} y),
\end{aligned}
$$

where $a(x)$ is a non-negative Borel measurable function, $b(x)$ is an $\mathbb{R}^{d}$-valued Borel measurable function, $c(x):=\left(c_{i j}(x)\right)_{1 \leq i, j \leq d}$ is a symmetric non-negative definite $d \times d$ matrix-valued Borel measurable function, and $v(x, \mathrm{~d} y)$ is a non-negative Borel kernel on $\mathbb{R}^{d} \times \mathcal{B}\left(\mathbb{R}^{d}\right)$, called the Lévy kernel, satisfying

$$
v(x,\{0\})=0 \text { and } \int_{\mathbb{R}^{d}}\left(1 \wedge|y|^{2}\right) v(x, \mathrm{~d} y)<\infty, \quad x \in \mathbb{R}^{d} .
$$

The quadruple $(a(x), b(x), c(x), v(x, \mathrm{~d} y))$ is called the Lévy quadruple of $\left.\mathcal{A}^{\infty}\right|_{C_{c}^{\infty}\left(\mathbb{R}^{d}\right)}$ (or of $q(x, \xi)$ ). Let us remark that local boundedness of $q(x, \xi)$ implies local boundedness of the corresponding $x$-coefficients, and vice versa (see [70, Lemma 2.1 and Remark 2.2]). In the sequel, we assume the following condition on the symbol $q(x, \xi)$ :

(LTP2) $q(x, 0)=a(x) \equiv 0$.

This condition is closely related to the conservativeness property of $\left\{X_{t}\right\}_{t \geq 0}$. Namely, under the assumption that the $x$-coefficients of $q(x, \xi)$ are uniformly bounded (which is certainly the case in the periodic setting), (LTP2) implies that $\left\{X_{t}\right\}_{t \geq 0}$ is conservative, that is, $\mathbb{P}_{x}\left(X_{t} \in \mathbb{R}^{d}\right)=1$ for all $t \geq 0$ and $x \in \mathbb{R}^{d}$ (see [69, Theorem 5.2]). Further, note that by combining Eqs. (1.4) and (1.5) with (LTP2), $\left.\mathcal{A}^{\infty}\right|_{C_{c}^{\infty}\left(\mathbb{R}^{d}\right)}$ takes the form Eq. (1.1). Conversely, if $\mathcal{L}: C_{c}^{\infty}\left(\mathbb{R}^{d}\right) \rightarrow C_{\infty}\left(\mathbb{R}^{d}\right)$ is a linear operator of the form Eq. (1.1) satisfying the so-called positive maximum principle $\left(\mathcal{L} f\left(x_{0}\right) \leq 0\right.$ for any $f \in C_{c}^{\infty}\left(\mathbb{R}^{d}\right)$ with $\left.f\left(x_{0}\right)=\sup _{x \in \mathbb{R}^{d}} f(x) \geq 0\right)$ and such that $(\lambda-\mathcal{L})\left(C_{c}^{\infty}\left(\mathbb{R}^{d}\right)\right)$ is dense in $C_{\infty}\left(\mathbb{R}^{d}\right)$ for some (or all) $\lambda>0$, then, according to the Hille-YosidaRay theorem, $\mathcal{L}$ is closable and the closure is the generator of a Feller semigroup. In particular, the corresponding Feller process is a Lévy-type process. In the case when $q(x, \xi)$ does not depend on the variable $x \in \mathbb{R}^{d},\left\{X_{t}\right\}_{t \geq 0}$ becomes a Lévy process, that is, a stochastic process with stationary and independent increments. Moreover, unlike Feller processes, every Lévy process is uniquely and completely characterized through its corresponding symbol (see [68, Theorems 7.10 and 8.1] and [14, Example 2.26]). According to this, it is not hard to check that every conservative Lévy process satisfies conditions (LTP1) and (LTP2) (see [68, Theorem 31.5]). Thus, the class of processes we consider in this article contains Lévy processes. Throughout this article, the symbol $\left\{X_{t}\right\}_{t \geq 0}$ denotes a Feller process satisfying conditions (LTP1) and (LTP2). Such a process is called a Lévy-type process (LTP). If $v(x, \mathrm{~d} y) \equiv 0$, $\left\{X_{t}\right\}_{t \geq 0}$ is called a diffusion process. Note that this definition agrees with the standard definition of (Feller-Dynkin) diffusions (see [65, Chapter III.2]). A typical example of a LTP is a solution to the following SDE

$$
\mathrm{d} X_{t}=\Phi\left(X_{t-}\right) \mathrm{d} Y_{t}, \quad X_{0}=x \in \mathbb{R}^{d},
$$


where $\Phi: \mathbb{R}^{d} \rightarrow \mathbb{R}^{d \times n}$ is locally Lipschitz continuous and bounded (which is not a restriction in the periodic setting), and $\left\{Y_{t}\right\}_{t \geq 0}$ is an $n$-dimensional Lévy process with symbol $q_{Y}(\xi)$. Namely, in [71, Theorems 3.1 and 3.5 and Corollary 3.3] it has been shown that the unique solution $\left\{X_{t}\right\}_{t \geq 0}$ to the SDE in Eq. (1.6) (which exists by standard arguments) is a LTP with symbol of the form $q(x, \xi)=q_{Y}\left(\Phi^{\prime}(x) \xi\right)$. Here, for a matrix $M, M^{\prime}$ denotes its transpose. Observe that the following SDE is a special case of Eq. (1.6),

$$
\mathrm{d} X_{t}=\Phi_{1}\left(X_{t}\right) \mathrm{d} t+\Phi_{2}\left(X_{t}\right) \mathrm{d} B_{t}+\Phi_{3}\left(X_{t-}\right) \mathrm{d} Z_{t}, \quad X_{0}=x \in \mathbb{R}^{d},
$$

where $\Phi_{1}: \mathbb{R}^{d} \rightarrow \mathbb{R}^{d}, \Phi_{2}: \mathbb{R}^{d} \rightarrow \mathbb{R}^{d \times p}$ and $\Phi_{3}: \mathbb{R}^{d} \rightarrow \mathbb{R}^{d \times q}$, with $p+q=$ $n-1$, are locally Lipschitz continuous and bounded, $\left\{B_{t}\right\}_{t \geq 0}$ is a $p$-dimensional Brownian motion, and $\left\{Z_{t}\right\}_{t \geq 0}$ is a $q$-dimensional pure-jump Lévy process (that is, a Lévy process determined by a Lévy triplet of the form $\left.\left(0,0, v_{Z}(\mathrm{~d} y)\right)\right)$. Namely, set $\Phi(x)=\left(\Phi_{1}(x), \Phi_{2}(x), \Phi_{3}(x)\right)$ for any $x \in \mathbb{R}^{d}$, and $Y_{t}=\left(t, B_{t}, Z_{t}\right)^{\prime}$ for $t \geq 0$. For more on Lévy-type processes we refer the readers to the monograph [14].

LTPs with periodic coefficients. Let $\tau=\left(\tau_{1}, \ldots, \tau_{d}\right) \in(0, \infty)^{d}$ be fixed, and let $\tau \mathbb{Z}^{d}:=\tau_{1} \mathbb{Z} \times \cdots \times \tau_{d} \mathbb{Z}$. For $k=\left(k_{1}, \ldots, k_{d}\right) \in \mathbb{Z}^{d}$ define $\tau \odot k:=\left(\tau_{1} k_{1}, \ldots, \tau_{d} k_{d}\right)$, and for $x \in \mathbb{R}^{d}$ define

$$
x_{\tau}:=\left\{y \in \mathbb{R}^{d}: x-y \in \tau \mathbb{Z}^{d}\right\} \text { and } \mathbb{R}^{d} / \tau \mathbb{Z}^{d}:=\left\{x_{\tau}: x \in \mathbb{R}^{d}\right\} .
$$

In the sequel, we denote $\mathbb{T}_{\tau}^{d}=\mathbb{R}^{d} / \tau \mathbb{Z}^{d}$. Clearly, $\mathbb{T}_{\tau}^{d}$ is obtained by identifying the opposite faces of $[0, \tau]:=\left[0, \tau_{1}\right] \times \cdots \times\left[0, \tau_{d}\right]$. Let $\Pi_{\tau}: \mathbb{R}^{d} \rightarrow \mathbb{T}_{\tau}^{d}, \Pi_{\tau}(x):=x_{\tau}$, be the covering map. A function $f: \mathbb{R}^{d} \rightarrow \mathbb{R}$ is called $\tau$-periodic if

$$
f(x+\tau \odot k)=f(x), \quad x \in \mathbb{R}^{d}, k \in \mathbb{Z}^{d} .
$$

Clearly, every $\tau$-periodic function $f(x)$ is completely and uniquely determined by its restriction $\left.f\right|_{[0, \tau]}(x)$ to $[0, \tau]$, and since $\left.f\right|_{[0, \tau]}(x)$ assumes the same value on opposite faces of $[0, \tau]$, it can be identified by a function $f_{\tau}: \mathbb{T}_{\tau}^{d} \rightarrow \mathbb{R}$ given with $f_{\tau}\left(x_{\tau}\right)=f(x)$. For notational convenience, we will often omit the subscript $\tau$ and simply write $x$ instead of $x_{\tau}$, and $f(x)$ instead of $f_{\tau}(x)$.

Let now $\left\{X_{t}\right\}_{t \geq 0}$ be a LTP with semigroup $\left\{P_{t}\right\}_{t \geq 0}$, symbol $q(x, \xi)$ and Lévy triplet $(b(x), c(x), v(x, \mathrm{~d} y))$, satisfying:

(C1) $x \mapsto q(x, \xi)$ is $\tau$-periodic for all $\xi \in \mathbb{R}^{d}$.

Directly from the Lévy-Khintchine formula it follows that (C1) is equivalent to the $\tau$-periodicity of the corresponding Lévy triplet $(b(x), c(x), v(x, \mathrm{~d} y))$, which in turn is equivalent to the $\tau$-periodicity of $x \mapsto \mathbb{P}_{x}\left(X_{t}-x \in \mathrm{d} y\right)$ (see [67, Section 4]). This immediately implies that $\left\{P_{t}\right\}_{t \geq 0}$ preserves the class of all bounded Borel measurable $\tau$-periodic functions, that is, the function $x \mapsto P_{t} f(x)$ is $\tau$-periodic for all $t \geq 0$ and all $\tau$-periodic $f \in B_{b}\left(\mathbb{R}^{d}\right)$. Now, together with this, a straightforward adaptation of [49, Proposition 3.8.3] entails that $\left\{\Pi_{\tau}\left(X_{t}\right)\right\}_{t \geq 0}$ is a Markov process on $\left(\mathbb{T}_{\tau}^{d}, \mathcal{B}\left(\mathbb{T}_{\tau}^{d}\right)\right)$ with 
positivity preserving contraction semigroup $\left\{P_{t}^{\tau}\right\}_{t \geq 0}$ (on the space $\left(B_{b}\left(\mathbb{T}_{\tau}^{d}\right),\|\cdot\|_{\infty}\right)$ ) given by

$$
P_{t}^{\tau} f(x):=\mathbb{E}_{x}^{\tau}\left[f\left(\Pi_{\tau}\left(X_{t}\right)\right)\right]=\int_{\mathbb{T}_{\tau}^{d}} f(y) \mathbb{P}_{x}^{\tau}\left(\Pi_{\tau}\left(X_{t}\right) \in \mathrm{d} y\right),
$$

for $t \geq 0, x \in \mathbb{T}_{\tau}^{d}$ and $f \in B_{b}\left(\mathbb{T}_{\tau}^{d}\right)$. Here, $\mathcal{B}\left(\mathbb{T}_{\tau}^{d}\right)$ stands for the Borel $\sigma$-algebra on $\mathbb{T}_{\tau}^{d}$ (with respect to the standard quotient topology), $B_{b}\left(\mathbb{T}_{\tau}^{d}\right)$ denotes the class of all bounded Borel measurable functions $f: \mathbb{T}_{\tau}^{d} \rightarrow \mathbb{R}$ (which can be identified with the class of all $\tau$-periodic bounded Borel measurable functions $f: \mathbb{R}^{d} \rightarrow \mathbb{R}$ ), and

$$
\mathbb{P}_{x}^{\tau}\left(\Pi_{\tau}\left(X_{t}\right) \in B\right):=\mathbb{P}_{z_{x}}\left(X_{t} \in \Pi_{\tau}^{-1}(B)\right), \quad t \geq 0, x \in \mathbb{T}_{\tau}^{d}, B \in \mathcal{B}\left(\mathbb{T}_{\tau}^{d}\right),
$$

with $z_{x}$ being an arbitrary point in $\Pi_{\tau}^{-1}(\{x\})$.

Further, assume that

(C2) $\left\{X_{t}\right\}_{t \geq 0}$ is strong Feller and open-set irreducible, that is, for any $t>0$, any $x \in \mathbb{R}^{d}$ and any non-empty open set $O \subseteq \mathbb{R}^{d}, \mathbb{P}_{x}\left(X_{t} \in O\right)>0$.

Clearly, (C2) automatically implies that the process $\left\{\Pi_{\tau}\left(X_{t}\right)\right\}_{t \geq 0}$ is strong Feller and open-set irreducible, too. Hence, by employing [51, Remark 3.2] and [85, Theorem 1.1] we have proved the following.

Proposition 1.1. The process $\left\{\Pi_{\tau}\left(X_{t}\right)\right\}_{t \geq 0}$ admits a unique invariant probability measure $\pi(\mathrm{d} x)$, that is, a measure $\pi(\mathrm{d} x)$ satisfying

$$
\int_{\mathbb{T}_{\tau}^{d}} \mathbb{P}_{x}^{\tau}\left(\Pi_{\tau}\left(X_{t}\right) \in B\right) \pi(\mathrm{d} x)=\pi(B), \quad t \geq 0, B \in \mathcal{B}\left(\mathbb{T}_{\tau}^{d}\right),
$$

such that

$$
\sup _{x \in \mathbb{T}_{\tau}^{d}}\left\|\mathbb{P}_{x}^{\tau}\left(\Pi_{\tau}\left(X_{t}\right) \in \mathrm{d} y\right)-\pi(\mathrm{d} y)\right\|_{\mathrm{TV}} \leq \Gamma \mathrm{e}^{-\gamma t}, \quad t \geq 0
$$

for some $\gamma, \Gamma>0$, where $\|\cdot\|_{\mathrm{TV}}$ denotes the total variation norm on the space of signed measures on $\mathcal{B}\left(\mathbb{T}_{\tau}^{d}\right)$.

Remark 1.2. Alternatively, Proposition 1.1 is a consequence of [57, Theorems 3.2 and 8.1] and [83, Theorem 3.2], or [58, Theorem 6.1] and [83, Theorem 5.1] (by setting $V(x) \equiv 1$ and $c=d=1)$. Also, if instead of (C2) we assume

( $\widetilde{\mathrm{C} 2})\left\{X_{t}\right\}_{t \geq 0}$ admits a density function $p_{t}(x, y)$ (with respect to the Lebesgue measure) satisfying

(i) for any $t>0$, the function $(x, y) \mapsto p_{t}(x, y)$ is continuous on $\mathbb{R}^{d} \times \mathbb{R}^{d}$;

(ii) there is a non-empty open set $O \subseteq \mathbb{R}^{d}$ such that $p_{t}(x, y)>0$ for all $t>0$, $x \in \mathbb{R}^{d}$ and $y \in O$,

which guarantees that Döblin's irreducibility condition holds true (see [25, page 256]), then Proposition 1.1 follows from [10, Theorem 3.1]. 
Conditions (in terms of the Lévy triplet $(b(x), c(x), v(x, \mathrm{~d} y)))$ ensuring $(\mathbf{C 2})$ are discussed in Sect. 3.

The semimartingale nature of LTPs. As we have already commented, the problem of homogenization of an operator of the form Eq. (1.1) corresponding to a LTP is equivalent to the convergence of the corresponding family of LTPs in the path space endowed with the Skorohod $\mathrm{J}_{1}$ topology (see [14, Theorem 7.1]). According to [70, Lemma 3.2], $\left\{X_{t}\right\}_{t \geq 0}$ is a $\mathbb{P}_{x}$ - semimartingale (with respect to the natural filtration) for any $x \in \mathbb{R}^{d}$. Therefore, in order to show this convergence, our aim is to employ [43, Theorem VIII.2.17] which states that a sequence of semimartingales converges in the path space endowed with the Skorohod $\mathrm{J}_{1}$ topology to a process with independent increments if the corresponding semimartingale characteristics converge in probability.

Let us now recall the notion of characteristics of a semimartingale (see [43]). Let $\left(\Omega, \mathcal{F},\left\{\mathcal{F}_{t}\right\}_{t \geq 0}, \mathbb{P},\left\{S_{t}\right\}_{t \geq 0}\right)$, denoted by $\left\{S_{t}\right\}_{t \geq 0}$ in the sequel, be a $d$-dimensional semimartingale, and let $h: \mathbb{R}^{d} \rightarrow \mathbb{R}^{d}$ be a truncation function (that is, a bounded Borel measurable function which satisfies $h(x)=x$ in a neighborhood of the origin). Define

$$
\check{S}(h)_{t}:=\sum_{s \leq t}\left(\Delta S_{s}-h\left(\Delta S_{S}\right)\right) \text { and } S(h)_{t}:=S_{t}-\check{S}(h)_{t}, \quad t \geq 0,
$$

where the process $\left\{\Delta S_{t}\right\}_{t \geq 0}$ is defined by $\Delta S_{t}:=S_{t}-S_{t-}$ and $\Delta S_{0}:=S_{0}$. The process $\left\{S(h)_{t}\right\}_{t \geq 0}$ is a special semimartingale, that is, it admits a unique decomposition

$$
S(h)_{t}=S_{0}+M(h)_{t}+B(h)_{t},
$$

where $\left\{M(h)_{t}\right\}_{t \geq 0}$ is a local martingale, and $\left\{B(h)_{t}\right\}_{t \geq 0}$ is a predictable process of bounded variation.

Definition 1.3. Let $\left\{S_{t}\right\}_{t \geq 0}$ be a semimartingale, and let $h: \mathbb{R}^{d} \longrightarrow \mathbb{R}^{d}$ be a truncation function. Furthermore, let $\left\{B(h)_{t}\right\}_{t \geq 0}$ be the predictable process of bounded variation appearing in Eq. (1.9), let $N(\omega, \mathrm{d} y, \mathrm{~d} s)$ be the compensator of the jump measure

$$
\mu(\omega, \mathrm{d} y, \mathrm{~d} s):=\sum_{s: \Delta S_{s}(\omega) \neq 0} \delta_{\left(\Delta S_{s}(\omega), s\right)}(\mathrm{d} y, \mathrm{~d} s)
$$

of the process $\left\{S_{t}\right\}_{t \geq 0}$, and let $\left.\left\{C_{t}\right\}_{t \geq 0}=\left\{\left(C_{t}^{i j}\right)_{1 \leq i, j \leq d}\right)\right\}_{t \geq 0}$ be the quadratic covariation process for $\left\{S_{t}^{c}\right\}_{t \geq 0}$ (continuous martingale part of $\left\{S_{t}\right\}_{t \geq 0}$ ), that is, $C_{t}^{i j}=$ $\left\langle S_{t}^{i, c}, S_{t}^{j, c}\right\rangle$. Then $(B, C, N)$ is called the characteristics of the semimartingale $\left\{S_{t}\right\}_{t \geq 0}$ (relative to $h(x)$ ). In addition, by defining $\tilde{C}(h)_{t}^{i j}:=\left\langle M(h)_{t}^{i}, M(h)_{t}^{j}\right\rangle, i, j=1, \ldots, d$, where $\left\{M(h)_{t}\right\}_{t \geq 0}$ is the local martingale appearing in Eq. (1.9), $(B, \tilde{C}, N)$ is called the modified characteristics of the semimartingale $\left\{S_{t}\right\}_{t \geq 0}$ (relative to $h(x)$ ). 
Now, according to [70, Theorem 3.5] and [43, Proposition II.2.17] we see that the (modified) characteristics of a LTP $\left\{X_{t}\right\}_{t \geq 0}$ (with respect to a truncation function $h(x)$ ) are given by

$$
\begin{aligned}
B(h)_{t}^{i} & =\int_{0}^{t} b_{i}\left(X_{s}\right) \mathrm{d} s+\int_{0}^{t} \int_{\mathbb{R}^{d}}\left(h_{i}(y)-y_{i} \mathbb{1}_{B_{1}(0)}(y)\right) v\left(X_{s}, \mathrm{~d} y\right) \mathrm{d} s, \\
C_{t}^{i j} & =\int_{0}^{t} c_{i j}\left(X_{s}\right) \mathrm{d} s, \\
N(\mathrm{~d} y, \mathrm{~d} s) & =v\left(X_{s}, \mathrm{~d} y\right) \mathrm{d} s, \\
\tilde{C}(h)_{t}^{i j} & =\int_{0}^{t} c_{i j}\left(X_{s}\right) \mathrm{d} s+\int_{0}^{t} \int_{\mathbb{R}^{d}} h_{i}(y) h_{j}(y) v\left(X_{s}, \mathrm{~d} y\right) \mathrm{d} s,
\end{aligned}
$$

for $t \geq 0$ and $i, j=1, \ldots, d$.

In the sequel, we assume that $\left\{X_{t}\right\}_{t \geq 0}$ admits "small jumps" only, that is,

(C3) $\sup _{x \in \mathbb{R}^{d}} \int_{\mathbb{R}^{d}}|y|^{2} v(x, \mathrm{~d} y)<\infty$.

As a direct consequence of (C3) and [43, Proposition II.2.29] we see that $\left\{X_{t}\right\}_{t \geq 0}$ itself is a special semimartingale, and for the truncation function we can take $h(x)=x$. In particular, if $v(x, \mathrm{~d} y)$ is also symmetric for every $x \in \mathbb{R}^{d}$, the first characteristic $B(h)_{t}^{i}$ equals to $\int_{0}^{t} b_{i}\left(X_{S}\right) \mathrm{d} s$ for $t \geq 0$ and $i=1, \ldots, d$.

Observe next that $\left\{X_{t}\right\}_{t \geq 0}$ is a Hunt process (since it is Feller). Thus, $\left\{X_{t}\right\}_{t \geq 0}$ is an Itô process in the sense of [22] (a semimartngale Hunt process with characteristics of the form as above). Now, [22, Theorem 3.33] asserts that there exist a suitable enlargement of the stochastic basis $\left(\Omega, \mathcal{F},\left\{\mathbb{P}_{x}\right\}_{x \in \mathbb{R}^{d}},\left\{\mathcal{F}_{t}\right\}_{t \geq 0},\left\{\theta_{t}\right\}_{t \geq 0}\right)$, say $\left(\widetilde{\Omega}, \widetilde{\mathcal{F}},\left\{\widetilde{\mathbb{P}}_{x}\right\}_{x \in \mathbb{R}^{d}},\left\{\widetilde{\mathcal{F}}_{t}\right\}_{t \geq 0},\left\{\widetilde{\theta}_{t}\right\}_{t \geq 0}\right)$, supporting a $d$-dimensional Brownian motion $\left\{\tilde{W}_{t}\right\}_{t \geq 0}$ and a Poisson random measure $\tilde{\mu}(\cdot, \mathrm{d} z, \mathrm{~d} s)$ on $\mathcal{B}(\mathbb{R}) \otimes \mathcal{B}([0, \infty))$ with compensator $\tilde{v}(\mathrm{~d} z) \mathrm{d} s$, such that $\left\{X_{t}\right\}_{t \geq 0}$ is a solution to the following stochastic differential equation

$$
\begin{aligned}
X_{t}= & +\int_{0}^{t} b\left(X_{s}\right) \mathrm{d} s+\int_{0}^{t} \tilde{\sigma}\left(X_{s}\right) \mathrm{d} \tilde{W}_{s} \\
& +\int_{0}^{t} \int_{\mathbb{R}} k\left(X_{s-}, z\right) \mathbb{1}_{\left\{u:\left|k\left(X_{s-}, u\right)\right|<1\right\}}(z)(\tilde{\mu}(\cdot, \mathrm{d} z, \mathrm{~d} s)-\tilde{v}(\mathrm{~d} z) \mathrm{d} s) \\
& +\int_{0}^{t} \int_{\mathbb{R}} k\left(X_{s-}, z\right) \mathbb{1}_{\left\{u:\left|k\left(X_{s-}, u\right)\right| \geq 1\right\}}(z) \tilde{\mu}(\cdot, \mathrm{d} z, \mathrm{~d} s),
\end{aligned}
$$

where $\tilde{\sigma}(x)$ is a $d \times d$ matrix-valued Borel measurable function such that $\tilde{\sigma}(x)^{\prime} \tilde{\sigma}(x)=$ $c(x)$ for any $x \in \mathbb{R}^{d}, \tilde{v}(\mathrm{~d} z)$ is any given $\sigma$-finite non-finite and non-atomic measure on $\mathcal{B}(\mathbb{R})$, and $k: \mathbb{R}^{d} \times \mathbb{R} \rightarrow \mathbb{R}^{d}$ is a Borel measurable function satisfying

$$
\mu(\cdot, \mathrm{d} y, \mathrm{~d} s)=\tilde{\mu}\left(\cdot,\left\{(z, u) \in \mathbb{R} \times[0, \infty):\left(k\left(X_{u-}, z\right), u\right) \in(\mathrm{d} y, \mathrm{~d} s)\right\}\right),
$$

and

$$
v(x, \mathrm{~d} y)=\tilde{v}(\{z \in \mathbb{R}: k(x, z) \in \mathrm{d} y\}) .
$$


Thus, due to this and (C3) we have that

$$
\begin{aligned}
X_{t}=x & +\int_{0}^{t} b\left(X_{s}\right) \mathrm{d} s+\int_{0}^{t} \tilde{\sigma}\left(X_{s}\right) \mathrm{d} \tilde{W}_{s} \\
& +\int_{0}^{t} \int_{\mathbb{R}} k\left(X_{s}, z\right) \mathbb{1}_{\left\{u:\left|k\left(X_{s}, u\right)\right| \geq 1\right\}}(z) \tilde{v}(\mathrm{~d} z) \mathrm{d} s \\
& +\int_{0}^{t} \int_{\mathbb{R}} k\left(X_{s-}, z\right)(\tilde{\mu}(\cdot, \mathrm{d} z, \mathrm{~d} s)-\tilde{v}(\mathrm{~d} z) \mathrm{d} s) .
\end{aligned}
$$

From this equation we also read the unique special semimartingale decomposition of $\left\{X_{t}\right\}_{t \geq 0}$.

Main result. Before stating the main result of this article, we introduce some notation we need. Denote by $C_{b}^{k}\left(\mathbb{R}^{d}\right)$ with $k \in \mathbb{N}_{0}:=\{0,1,2, \ldots\}$ the space of $k$ times differentiable functions such that all derivatives up to order $k$ are bounded. This space is a Banach space endowed with the norm $\|f\|_{k}:=\sum_{m:|m| \leq k}\left\|D^{m} f\right\|_{\infty}$, where $m=$ $\left(m_{1}, \ldots, m_{d}\right) \in \mathbb{N}_{0}^{d},|m|:=m_{1}+\cdots+m_{d}$, and $D^{m}:=\partial^{m_{1}} \ldots \partial^{m_{d}}$. Denote also $C_{b}^{\infty}\left(\mathbb{R}^{d}\right):=\cap_{k \in \mathbb{N}_{0}} C_{b}^{k}\left(\mathbb{R}^{d}\right)$. Further, a function $\phi:(0,1] \rightarrow(0, \infty)$ is said to be almost increasing if there is a constant $\underline{\kappa} \in(0,1]$ such that $\underline{\kappa} \phi(r) \leq \phi(R)$ for all $r, R \in(0,1]$ with $r \leq R$. Analogously, $\phi:(0,1] \rightarrow(0, \infty)$ is said to be almost decreasing if there is a constant $\bar{\kappa} \in[1, \infty)$ such that $\phi(R) \leq \bar{\kappa} \phi(r)$ for all $r, R \in$ $(0,1]$ with $r \leq R$. Let now $\psi:(0,1] \rightarrow[0, \infty)$ be such that $\psi(1)=1$ and $\lim _{r \rightarrow 0} \psi(r)=0$. For $f \in C_{b}\left(\mathbb{R}^{d}\right)$ and $j \in \mathbb{N}_{0}$, define

$$
[f]_{-j, \psi}:=\sup _{x \in \mathbb{R}^{d}} \sup _{h \in \bar{B}_{1}(0) \backslash\{0\}} \frac{|f(x+h)-f(x)|}{\psi(|h|)|h|^{-j}},
$$

where $\bar{B}_{r}(x)$ stands for the (topologically) closed ball of radius $r$ around $x \in \mathbb{R}^{d}$. Also, let

$$
\begin{aligned}
& m_{\psi}:=\sup \left\{\alpha \in \mathbb{R}: r \mapsto \psi(r) / r^{\alpha} \text { is almost increasing in }(0,1]\right\}, \\
& M_{\psi}:=\inf \left\{\alpha \in \mathbb{R}: r \mapsto \psi(r) / r^{\alpha} \text { is almost decreasing in }(0,1]\right\} .
\end{aligned}
$$

According to [12, Theorem 2.2.2], $m_{\psi} \leq M_{\psi}$. If $m_{\psi}>0$, we call $\psi(r)$ the Hölder exponent. In this case, if $m_{\psi} \in(k, k+1]$ for some $k \in \mathbb{N}_{0}$, define

$$
C_{b}^{\psi}\left(\mathbb{R}^{d}\right):=\left\{f \in C_{b}^{k}\left(\mathbb{R}^{d}\right):\left[D^{m} f\right]_{-k, \psi}<\infty \text { for }|m|=k\right\} .
$$

This space is called a generalized Hölder space, and it is a normed vector space with the norm

$$
\|f\|_{\psi}:=\|f\|_{k}+\sum_{m:|m|=k}\left[D^{m} f\right]_{-k, \psi},
$$

(see [6]). Observe that the product of two Hölder exponents is a Hölder exponent, and that if $m_{\psi} \in(k, k+1]$ for some $k \in \mathbb{N}_{0}$ then $C_{b}^{k+1}\left(\mathbb{R}^{d}\right) \subsetneq C_{b}^{\psi}\left(\mathbb{R}^{d}\right) \subsetneq C_{b}^{k}\left(\mathbb{R}^{d}\right)$. In 
particular, when $\psi(r)=r^{\gamma}$ for some $\gamma>0, C_{b}^{\psi}\left(\mathbb{R}^{d}\right)$ becomes the classical Hölder space of order $\gamma$ (usually denoted by $C_{b}^{\gamma}\left(\mathbb{R}^{d}\right)$ ), which is a Banach space together with the above-defined norm (which we denote by $\|\cdot\|_{\gamma}$ ). Since $f \leftrightarrow f_{\tau}$ gives a one-to-one correspondence between $\left\{f: \mathbb{R}^{d} \rightarrow \mathbb{R}: f\right.$ is $\tau$-periodic $\}$ and $\left\{f_{\tau}: \mathbb{T}_{\tau}^{d} \rightarrow \mathbb{R}\right\}$, in an analogous way we define $C^{k}\left(\mathbb{T}_{\tau}^{d}\right)$ and $C^{\psi}\left(\mathbb{T}_{\tau}^{d}\right)$.

We are now in position to state the main result of this article, the proof of which is given in Sect. 2 .

Theorem 1.4. Let $\left\{X_{t}\right\}_{t \geq 0}$ be a d-dimensional LTP with semigroup $\left\{P_{t}\right\}_{t \geq 0}$, symbol $q(x, \xi)$ and Lévy triplet $(b(x), c(x), v(x, \mathrm{~d} y))$, satisfying $(\boldsymbol{C 1}),(\boldsymbol{C 2}),(\boldsymbol{C 3})$ and

(C4) $x \mapsto b^{*}(x):=b(x)+\int_{B_{1}^{c}(0)} y v(x, \mathrm{~d} y)$ is of class $C_{b}^{\psi}\left(\mathbb{R}^{d}\right)$ for some Hölder exponent $\psi(r)$, and

(i) for some $t_{0}>0$, any $t \in\left(0, t_{0}\right]$ and any $\tau$-periodic $f \in C_{b}\left(\mathbb{R}^{d}\right)$,

$$
\left\|P_{t} f\right\|_{\psi} \leq C(t)\|f\|_{\infty}
$$

where $\int_{0}^{t_{0}} C(t) \mathrm{d} t<\infty$

(ii) for some $\lambda>0$ and any $\tau$-periodic $f \in C_{b}^{\psi}\left(\mathbb{R}^{d}\right)$ with $\int_{\mathbb{T}_{\tau}^{d}} f_{\tau}(x) \pi(\mathrm{d} x)=0$, the Poisson equation

$$
\lambda u-\mathcal{A}^{b} u=f
$$

admits a $\tau$-periodic solution $u_{\lambda, f} \in C_{b}^{\varphi \psi}\left(\mathbb{R}^{d}\right)$ for some Hölder exponent $\varphi(r)$. Then,

(a) the Poisson equation

$$
\mathcal{A}^{b} \beta=b^{*}-\overline{b^{*}}
$$

admits a $\tau$-periodic solution $\beta \in C_{b}^{\varphi \psi}\left(\mathbb{R}^{d}\right)$. Moreover, $\beta(x)$ is the unique solution in the class of continuous and periodic solutions to Eq. (1.12) satisfying $\int_{\mathbb{T}_{\tau}^{d}} \beta_{\tau}(x) \pi(\mathrm{d} x)=0$.

(b) in any of the following three cases

(1) $\beta \in C_{b}^{2}\left(\mathbb{R}^{d}\right)$ if $c(x) \not \equiv 0$;

(2) $m_{\varphi \psi}>1$ if $c(x) \equiv 0$ and

$$
\sup _{x \in \mathbb{R}^{d}} \int_{B_{1}(0)} \varphi(|y|) \psi(|y|) v(x, \mathrm{~d} y)<\infty ;
$$

(3) $\beta \in C_{b}^{1}\left(\mathbb{R}^{d}\right)$ if $c(x) \equiv 0$ and

$$
\sup _{x \in \mathbb{R}^{d}} \int_{B_{1}(0)}|y| v(x, \mathrm{~d} y)<\infty,
$$

for any initial distribution of $\left\{X_{t}\right\}_{t \geq 0}$,

$$
\left\{\varepsilon X_{\varepsilon^{-2} t}-\varepsilon^{-1} \overline{b^{*} t}\right\}_{t \geq 0} \stackrel{\varepsilon \rightarrow 0}{\Longrightarrow}\left\{W_{t}\right\}_{t \geq 0} .
$$


Here,

$$
\overline{b^{*}}:=\int_{\mathbb{T}_{\tau}^{d}} b^{*}(x) \pi(\mathrm{d} x),
$$

$\Rightarrow$ denotes the convergence in the space of càdlàg functions endowed with the Skorohod $\mathrm{J}_{1}$-topology, and $\left\{W_{t}\right\}_{t \geq 0}$ is a d-dimensional zero-drift Brownian motion determined by covariance matrix $\Sigma$ given in Eq. (1.3).

Under (C1), (C2) and the assumption that $b^{*} \in C_{b}\left(\mathbb{R}^{d}\right)$, in Lemma 2.1 below we show that Eq. 1.12 admits a $\tau$-periodic solution $\beta \in C_{b}\left(\mathbb{R}^{d}\right)$ (which is also unique in the class of continuous $\tau$-periodic solutions satisfying $\left.\int_{\mathbb{T}_{\tau}^{d}} \beta_{\tau}(x) \pi(\mathrm{d} x)=0\right)$. However, we require additional smoothness of $\beta(x)$ in order to apply Itô's formula (given in Proposition 2.2) in the proof of Theorem 1.4 (see Sect. 2 for details). This additional regularity is given through $(\mathbf{C 4})$ [together with $(\mathbf{C 1})$ and $(\mathbf{C 2})]$. Namely, under these assumptions, we show that $\beta \in C_{b}^{\varphi \psi}\left(\mathbb{R}^{d}\right)$. When $c(x) \not \equiv 0$ we require $\beta \in C_{b}^{2}\left(\mathbb{R}^{d}\right)$, and when $c(x) \equiv 0$ and $b(x) \not \equiv 0$ or $v(x, \mathrm{~d} y)$ is non-symmetric for some $x \in \mathbb{R}^{d}$ we only require $m_{\varphi \psi}>1$ or $\beta \in C_{b}^{1}\left(\mathbb{R}^{d}\right)$. When $b(x) \equiv 0$ and $\nu(x, \mathrm{~d} y)$ is symmetric for all $x \in \mathbb{R}^{d}$, as already commented, $\beta(x) \equiv 0$ and the assertion of the theorem follows without assuming $(\mathbf{C 4})$. In the pure-jump case (that is, when $c(x) \equiv 0)$, Eq. (1.13) suggests that the Hölder exponent $\varphi(r)$ depends on the behavior of $v(x, \mathrm{~d} y)$ on $B_{1}(0)$. For example, when

$$
\frac{\underline{\kappa}}{|y|^{d} \varphi(|y|)} \mathbb{1}_{B_{1}(0)}(y) \mathrm{d} y \leq \mathbb{1}_{B_{1}(0)}(y) v(x, \mathrm{~d} y) \leq \frac{\bar{\kappa}}{|y|^{d} \varphi(|y|)} \mathbb{1}_{B_{1}(0)}(y) \mathrm{d} y,
$$

for some $0<\underline{\kappa} \leq \bar{\kappa}<\infty$, Eq. (1.13) trivially holds true. Thus, we only require that $\beta \in C_{b}^{\varphi \psi}\left(\mathbb{R}^{d}\right)$ for some Hölder exponent $\psi(r)$ with $m_{\varphi \psi}>1$. Analogously, if Eq. (1.14) holds true, then we only require that $\beta \in C_{b}^{1}\left(\mathbb{R}^{d}\right)$. Observe that in the purejump case we do not require explicitly that $\beta \in C_{b}^{2}\left(\mathbb{R}^{d}\right)$. In this sense the assumption $u_{\lambda, f} \in C_{b}^{\varphi \psi}\left(\mathbb{R}^{d}\right)$ in (C4)(ii) is optimal. Namely, under Eq. (1.13) [resp. Eq. (1.14)], in Proposition 2.2 we show that when $m_{\varphi \psi}>1$ [resp. $\beta \in C_{b}^{1}\left(\mathbb{R}^{d}\right)$ ] we can apply Itô's formula to the process $\left\{\beta\left(X_{t}\right)\right\}_{t \geq 0}$.

Let us also remark that in the proof of Theorem 1.4 (a) we show that (C4)(i) [together with (C1)-(C3)] implies that $\beta \in C_{b}^{\psi}\left(\mathbb{R}^{d}\right)$. Hence, Eq. (1.15) holds true if $\psi(r)$ is such that either (1), (2) (with $\varphi(r) \equiv 1$ ) or (3) above is satisfied. If this is not the case, then we require an additional regularity of $\beta(x)$ (inherited from the semigroup) which is given through (C4)(ii).

Several examples of LTPs satisfying $(\mathbf{C 4})$ [and (C1)-(C3)] are presented in Sect. 3. In particular, if $\left\{X_{t}\right\}_{t \geq 0}$ is a diffusion process with $\tau$-periodic coefficients $b \in C_{b}^{\varepsilon}\left(\mathbb{R}^{d}\right)$ and $c \in C_{b}^{1+\varepsilon}\left(\mathbb{R}^{d}\right)$ for some $\varepsilon \in(0,1)$, and additionally $c(x)$ being positive definite, (C4) with $\psi(r)=r^{\varepsilon}$ and $\varphi(r)=r^{2}$ follows from [60, Theorem 2.1]. In particular, Theorem 1.4 generalizes the results from $[10,11]$ where periodic homogenization of a diffusion process with $\tau$-periodic coefficients $b \in C_{b}^{1}\left(\mathbb{R}^{d}\right)$ and $c \in C_{b}^{2}\left(\mathbb{R}^{d}\right)$, and $c(x)$ being positive definite, has been considered. 
If $\left\{X_{t}\right\}_{t \geq 0}$ is a LTP with diffusion and drift coefficients as above, and Lévy kernel satisfying Eq. (1.16) (and some additional regularity properties discussed in Sect. 3), then $(\mathbf{C 4})$ with $\psi(r)=r^{\varepsilon}$ and $\varphi(r)=r^{2}$ follows again from [60, Theorem 2.1].

If $\left\{X_{t}\right\}_{t \geq 0}$ is a pure-jump LTP with vanishing drift term and Lévy kernel satisfying Eq. (1.16) (and some additional regularity properties discussed in Sect. 3), (C4) holds true for any Hölder exponent $\psi(r)$ such that $\left[m_{\psi}, M_{\psi}\right] \subset(0,1)$ and $\left[m_{\varphi \psi}, M_{\varphi \psi}\right] \cap$ $\mathbb{N}=\emptyset$ (see Sect. 3).

Literature review. Our work relates to the active research on homogenization of integro-differential operators, and Markov processes with jumps. The work is highly motivated by the results in $[10,11,67]$ where, by employing probabilistic techniques, the authors considered periodic homogenization of the operator $\mathcal{L}$ with $v(x, \mathrm{~d} y) \equiv 0$ (that is, second-order elliptic operator in non-divergence form), and $\mathcal{L}$ with $b(x) \equiv 0$ and $v(x, \mathrm{~d} y)$ being symmetric for all $x \in \mathbb{R}^{d}$ (that is, integro-differential operator in the balanced form), respectively. In this article, we generalize both results by including the non-local part of the operator $\mathcal{L}$, as well as non-symmetries caused by the drift term $b(x)$ and the Lévy kernel $v(x, \mathrm{~d} y)$. In a closely related work [62], by using analytic techniques (the corrector method), the authors discuss periodic homogenization of the operator $\mathcal{L}$ with a convolution-type Lévy kernel, that is, $\mathcal{L}$ is determined by

$$
v(x, \mathrm{~d} y)=\lambda(x) \mu(x+y) a(y) \mathrm{d} y \quad \text { and } \quad b(x)=\int_{B_{1}(0)} y v(x, \mathrm{~d} y)
$$

with $\lambda(x)$ and $\mu(x)$ being measurable, $\tau$-periodic and such that $0<\underline{\kappa} \leq \lambda(x), \mu(x) \leq$ $\bar{\kappa}<\infty$ for all $x \in \mathbb{R}^{d}$, and $a(y) \geq 0$ being measurable and such that $0<\int_{\mathbb{R}^{d}}(1 \vee$ $\left.|y|^{2}\right) a(y) \mathrm{d} y<\infty$ and $a(y)=a(-y)$ for all $y \in \mathbb{R}^{d}$. The homogenized operator is again a second-order elliptic operator with constant coefficients. Observe that this case is not covered by Theorem 1.4 since finiteness of $v(x, \mathrm{~d} y)$ excludes regularity properties of the corresponding semigroup assumed in (C4).

There is a vast literature on homogenization of differential operators, mostly based on PDE methods. We refer the interested readers to [10,16,23,44,81] and the references therein. Results related to the problem of periodic homogenization of non-local operators (based on probabilistic techniques) were obtained in [31-33,35,37-39,82]. In all this works the focus is on the so-called stable-like operators (possibly with variable order), that is, on the case when $v(x, \mathrm{~d} y)$ admits "large jumps" of power-type:

$$
\frac{\underline{\kappa}}{|y|^{d+\bar{\alpha}}} \mathbb{1}_{B_{1}^{c}(0)}(y) \mathrm{d} y \leq \mathbb{1}_{B_{1}^{c}(0)}(y) v(x, \mathrm{~d} y) \leq \frac{\bar{\kappa}}{|y|^{d+\underline{\alpha}}} \mathbb{1}_{B_{1}^{c}(0)}(y) \mathrm{d} y, \quad x \in \mathbb{R}^{d},
$$

for some $0<\underline{\kappa} \leq \bar{\kappa}<\infty$ and $0<\underline{\alpha} \leq \bar{\alpha}<2$. In this case, by using subdiffusive scaling (which depends on the behavior of $v(x, \mathrm{~d} y)$ on $\left.B_{1}^{c}(0)\right)$, the homogenized operator is the infinitesimal generator of a stable Lévy process with the index of stability being equal to the power of the scaling factor. The problem of stochastic homogenization (that is, homogenization of operators with random coefficients) of this type of operators has been considered in [64]. PDE and other analytical approaches to 
the problem of periodic homogenization and stochastic homogenization of stable-like operators can be found in $[2-4,7,15,29,30,45,73,75,76]$.

Let us also remark that the class of processes considered in the present article constitute of both diffusion and pure-jump part, and the behavior of the homogenized process depends on both of them. This makes the approach to this problem more subtle since we need to take care of diffusion processes, diffusion processes with jumps and pure jump processes, simultaneously.

\section{Proof of Theorem 1.4}

Throughout this section we assume that $\left\{X_{t}\right\}_{t \geq 0}$ is a $d$-dimensional LTP with semigroup $\left\{P_{t}\right\}_{t \geq 0}$, symbol $q(x, \xi)$ and Lévy triplet $(b(x), c(x), v(x, \mathrm{~d} y))$, satisfying (C1)(C4). A crucial step in the proof is an application of Itô's formula. In order to justify this step, we first discuss regularity of a solution to the Poisson equation Eq. (1.12).

Solution to the Poisson equation Eq. (1.12). Observe first that for any $f_{\tau} \in B_{b}\left(\mathbb{T}_{\tau}^{d}\right)$ with $\int_{\mathbb{T}_{\tau}^{d}} f_{\tau}(x) \pi(\mathrm{d} x)=0$, Proposition 1.1 implies that

$$
\left\|P_{t}^{\tau} f_{\tau}\right\|_{\infty} \leq \Gamma \mathrm{e}^{-\gamma t}\left\|f_{\tau}\right\|_{\infty}, \quad t \geq 0
$$

In particular,

$$
\left\|\int_{0}^{\infty} P_{t}^{\tau} f_{\tau} \mathrm{d} t\right\|_{\infty} \leq \frac{\Gamma}{\gamma}\left\|f_{\tau}\right\|_{\infty}<\infty .
$$

Therefore, the zero-resolvent

$$
R^{\tau} f_{\tau}(x):=\int_{0}^{\infty} P_{t}^{\tau} f_{\tau}(x) \mathrm{d} t, \quad x \in \mathbb{T}_{\tau}^{d},
$$

is well defined, and

$$
\int_{\mathbb{T}_{\tau}^{d}} R^{\tau} f_{\tau}(x) \pi(\mathrm{d} x)=0 .
$$

According to [69, Corollary 3.4], $\left\{X_{t}\right\}_{t \geq 0}$ is a $C_{b}$-Feller process. Thus, $\left\{\Pi_{\tau}\left(X_{t}\right)\right\}_{t \geq 0}$ is also $C_{b}$-Feller, and $R^{\tau} f_{\tau} \in C\left(\mathbb{T}_{\tau}^{d}\right)$ for every $f_{\tau} \in C\left(\mathbb{T}_{\tau}^{d}\right)$ satisfying $\int_{\mathbb{T}_{\tau}^{d}} f_{\tau}(x) \pi(\mathrm{d} x)$ $=0$. Since $\mathbb{T}_{\tau}^{d}$ is compact, $\left\{\Pi_{\tau}\left(X_{t}\right)\right\}_{t \geq 0}$ is a Feller process. Denote the corresponding Feller generator by $\left(\mathcal{A}_{\tau}^{\infty}, \mathcal{D}_{\mathcal{A}_{\tau}^{\infty}}\right)$. Clearly, for any $f_{\tau} \in \mathcal{D}_{\mathcal{A}_{\tau}^{\infty}}$ (which is by definition continuous), and its $\tau$-periodic extension $f(x)$, it holds that $f \in \mathcal{D}_{\mathcal{A}^{b}}$ and $\mathcal{A}_{\tau}^{\infty} f_{\tau}=$ $\mathcal{A}^{b} f$. It is clear now that $R^{\tau} f_{\tau} \in \mathcal{D}_{\mathcal{A}_{\tau}^{\infty}}$ for any $f_{\tau} \in C\left(\mathbb{T}_{\tau}^{d}\right)$ with $\int_{\mathbb{T}_{\tau}^{d}} f_{\tau}(x) \pi(\mathrm{d} x)=0$.

Now, we turn to the Poisson equation Eq. (1.12). Denote by $b_{\tau}^{*}(x)$ the restriction of $b^{*}(x)$ to $\mathbb{T}_{\tau}^{d}$, and set $\overline{b_{\tau}^{*}}=\int_{\mathbb{T}_{\tau}^{d}} b_{\tau}^{*}(x) \pi(\mathrm{d} x)$. By assumption $b_{\tau}^{*} \in C^{\psi}\left(\mathbb{T}_{\tau}^{d}\right)$. Define now $\beta_{\tau}(x):=-R^{\tau}\left(b_{\tau}^{*}(\cdot)-\bar{b}_{\tau}^{*}\right)(x)$ for any $x \in \mathbb{T}_{\tau}^{d}$. According to the argument above, we immediately get the following. 
Lemma 2.1. The $\tau$-periodic extension $\beta(x)$ of $\beta_{\tau}(x)$ is continuous and satisfies Eq. (1.12). Moreover, $\beta(x)$ is the unique solution in the class of continuous and $\tau$-periodic solutions to Eq. (1.12) satisfying $\int_{\mathbb{T}_{\tau}^{d}} \beta_{\tau}(x) \pi(\mathrm{d} x)=0$.

Proof. We only need to prove uniqueness. Let $\bar{\beta}(x)$ be another continuous and $\tau$ periodic solution to Eq. (1.12) satisfying $\int_{\mathbb{T}_{\tau}^{d}} \bar{\beta}_{\tau}(x) \pi(\mathrm{d} x)=0$. Then, $\mathcal{A}^{b}(\beta-\bar{\beta})(x) \equiv$ 0. In particular, according to [28, Proposition 4.1.7],

$$
(\beta-\bar{\beta})(x)=\mathbb{E}_{x}\left[(\beta-\bar{\beta})\left(X_{t}\right)\right]=\mathbb{E}_{x_{\tau}}^{\tau}\left[\left(\beta_{\tau}-\bar{\beta}_{\tau}\right)\left(\Pi_{\tau}\left(X_{t}\right)\right)\right], \quad x \in \mathbb{R}^{d}, t \geq 0 .
$$

By letting now $t \rightarrow \infty$, it follows from Propostion 1.1 that $(\beta-\bar{\beta})(x) \equiv 0$, which proves the assertion.

Observe that in Lemma 2.1 we only used the fact that $b_{\tau}^{*} \in C\left(\mathbb{T}_{\tau}^{d}\right)$. In the sequel, we discuss additional smoothness of $\beta(x)$.

Proof of Theorem 1.4 (a). We first claim that for any $\tau$-periodic $f \in C_{b}\left(\mathbb{R}^{d}\right)$ such that $\int_{\mathbb{T}_{\tau}^{d}} f_{\tau}(x) \pi(\mathrm{d} x)=0, R^{\tau} f_{\tau} \in C^{\psi}\left(\mathbb{T}_{\tau}^{d}\right)$. Indeed, by (C4)(i), we have

$$
\int_{0}^{t_{0}}\left\|P_{t}^{\tau} f_{\tau}\right\|_{\psi} \mathrm{d} t \leq\left\|f_{\tau}\right\|_{\infty} \int_{0}^{t_{0}} C(t) \mathrm{d} t<\infty .
$$

Also, since for any $t>0$ and any $f_{\tau} \in C\left(\mathbb{T}_{\tau}^{d}\right)$ with $\int_{\mathbb{T}_{\tau}^{d}} f_{\tau}(x) \pi(\mathrm{d} x)=0, P_{t}^{\tau} f_{\tau} \in$ $C\left(\mathbb{T}_{\tau}^{d}\right)$ and $\int_{\mathbb{T}_{\tau}^{d}} P_{t}^{\tau} f_{\tau}(x) \pi(\mathrm{d} x)=0,(\mathbf{C 4})(\mathrm{i})$ and Proposition 1.1 imply that

$$
\begin{aligned}
\int_{t_{0}}^{\infty}\left\|P_{t}^{\tau} f_{\tau}\right\|_{\psi} \mathrm{d} t & \leq C\left(t_{0}\right) \int_{t_{0}}^{\infty}\left\|P_{t-t_{0}}^{\tau} f_{\tau}\right\|_{\infty} \mathrm{d} t \\
& \leq \Gamma C\left(t_{0}\right)\left\|f_{\tau}\right\|_{\infty} \int_{t_{0}}^{\infty} \mathrm{e}^{-\lambda\left(t-t_{0}\right)} \mathrm{d} t<\infty
\end{aligned}
$$

Combining both estimates above with the fact that $R^{\tau} f_{\tau}=\int_{0}^{\infty} P_{t}^{\tau} f_{\tau} \mathrm{d} t$, we get $R^{\tau} f_{\tau} \in C^{\psi}\left(\mathbb{T}_{\tau}^{d}\right)$.

Finally, for $\lambda>0$ let $R_{\lambda}^{\tau}$ be the $\lambda$-resolvent of $\left\{\Pi_{\tau}\left(X_{t}\right)\right\}_{t \geq 0}$. Clearly, for any $\tau$-periodic $f \in C_{b}^{\psi}\left(\mathbb{R}^{d}\right)$ satisfying $\int_{\mathbb{T}_{\tau}^{d}} f_{\tau}(x) \pi(\mathrm{d} x)=0$, the $\tau$-periodic extension of $R_{\lambda}^{\tau} f_{\tau}(x)$ (say $\bar{u}_{\lambda, f}(x)$ ) is a solution to Eq. (1.11). Observe next that necessarily $u_{\lambda, f}(x)=\bar{u}_{\lambda, f}(x)$ for all $x \in \mathbb{R}^{d}$. Namely, since $\bar{u}_{\lambda, f} \in C_{b}\left(\mathbb{R}^{d}\right)$, and by (1.11),

$$
\begin{aligned}
u_{\lambda, f}(x)-\bar{u}_{\lambda, f}(x)= & \mathrm{e}^{-\lambda t} \mathbb{E}_{x}\left[\left(u_{\lambda, f}-\bar{u}_{\lambda, f}\right)\left(X_{t}\right)\right] \\
& -\int_{0}^{t} \mathrm{e}^{-\lambda s} \mathbb{E}_{x}\left[\mathcal{A}^{b}\left(u_{\lambda, f}-\bar{u}_{\lambda, f}\right)\left(X_{s}\right)-\lambda\left(u_{\lambda, f}-\bar{u}_{\lambda, f}\right)\left(X_{s}\right)\right] \mathrm{d} s \\
= & \mathrm{e}^{-\lambda t} \mathbb{E}_{x}\left[\left(u_{\lambda, f}-\bar{u}_{\lambda, f}\right)\left(X_{t}\right)\right], \quad x \in \mathbb{R}^{d}, t \geq 0,
\end{aligned}
$$

by letting $t \rightarrow \infty$ the assertion follows. Thus, since $b_{\tau}^{*} \in C_{b}^{\psi}\left(\mathbb{T}_{\tau}^{d}\right)$, from the resolvent identity

$$
R^{\tau}\left(b_{\tau}^{*}(\cdot)-\bar{b}_{\tau}^{*}\right)(x)=R_{\lambda}^{\tau}\left(\left(b_{\tau}^{*}(\cdot)-\bar{b}_{\tau}^{*}\right)+\lambda R^{\tau}\left(b_{\tau}^{*}(\cdot)-\bar{b}_{\tau}^{*}\right)\right)(x)
$$


and (C4)(ii), we conclude the result.

In Theorem $1.4(\mathrm{~b})$ we require that $\beta \in C_{b}^{2}\left(\mathbb{R}^{d}\right)$ if $c(x) \not \equiv 0$, and that $m_{\varphi \psi}>1$ [resp. $\left.\beta \in C_{b}^{1}\left(\mathbb{R}^{d}\right)\right]$ if $c(x) \equiv 0$ and Eq. (1.13) [resp. Eq. (1.14)] holds true. In the following proposition we slightly generalize [63, Lemma 4.2] (see also [27]), and prove Itô's formula for a pure-jump LTP with respect to a not necessarily twice continuously differentiable function.

Proposition 2.2. Assume that $\left\{X_{t}\right\}_{t \geq 0}$ is pure-jump (that is, $c(x) \equiv 0$ ) and that there is a Hölder exponent $\phi(r)$ with $m_{\phi}>1$ such that

$$
\sup _{x \in \mathbb{R}^{d}} \int_{B_{1}(0)} \phi(|y|) v(x, \mathrm{~d} y)<\infty .
$$

Then, it holds that

$$
\begin{aligned}
f\left(X_{t}\right)= & f\left(X_{0}\right)+\int_{0}^{t}\left\langle\nabla f\left(X_{s}\right), b^{*}\left(X_{s}\right)\right\rangle \mathrm{d} s \\
& +\int_{0}^{t} \int_{\mathbb{R}^{d}}\left(f\left(X_{s-}+k\left(X_{s-}, z\right)\right)-f\left(X_{s-}\right)\right)(\tilde{\mu}(\cdot, \mathrm{d} z, \mathrm{~d} s)-\tilde{v}(\mathrm{~d} z) \mathrm{d} s) \\
& +\int_{0}^{t} \int_{\mathbb{R}^{d}}\left(f\left(X_{s}+k\left(X_{s}, z\right)\right)-f\left(X_{s}\right)-\left\langle\nabla f\left(X_{s}\right), k\left(X_{s}, z\right)\right\rangle\right) \tilde{v}(\mathrm{~d} z) \mathrm{d} s,
\end{aligned}
$$

for all $t \geq 0$ and all $f \in C_{b}^{\phi}\left(\mathbb{R}^{d}\right)$, where $b^{*}(x)=b(x)+\int_{B_{1}^{c}(0)} y v(x, \mathrm{~d} y)$. In addition, if Eq. (1.14) holds true, then the above relation holds for any $f \in C_{b}^{1}\left(\mathbb{R}^{d}\right)$.

Proof. Without loss of generality, assume that $m_{\phi} \in(1,2]$. We follow the proof of [63, Lemma 4.2]. Let $f \in C_{b}^{\phi}\left(\mathbb{R}^{d}\right)$, and let $\chi \in C_{c}^{\infty}\left(\mathbb{R}^{d}\right), 0 \leq \chi \leq 1$, be such that $\int_{\mathbb{R}^{d}} \chi(x) \mathrm{d} x=1$. For $n \in \mathbb{N}$ define $\chi_{n}(x):=n^{d} \chi(n x)$, and $f_{n}(x):=\left(\chi_{n} * f\right)(x)$, where $*$ stands for the standard convolution operator. Clearly, $\left\{f_{n}\right\}_{n \in \mathbb{N}} \subset C_{b}^{\infty}\left(\mathbb{R}^{d}\right)$, $\left\|f_{n}\right\|_{\phi} \leq\|f\|_{\phi}$ for $n \in \mathbb{N}, \lim _{n \rightarrow \infty} f_{n}(x)=f(x)$ and $\lim _{n \rightarrow \infty} \nabla f_{n}(x)=\nabla f(x)$ for all $x \in \mathbb{R}^{d}$. Next, by employing Itô's formula and Eq. (1.10) we have that

$$
\begin{aligned}
f_{n}\left(X_{t}\right)= & f_{n}\left(X_{0}\right)+\int_{0}^{t}\left\langle\nabla f_{n}\left(X_{s}\right), b\left(X_{s}\right)\right\rangle \mathrm{d} s \\
& +\int_{0}^{t} \int_{\mathbb{R}}\left\langle\nabla f_{n}\left(X_{s}\right), k\left(X_{s}, z\right)\right\rangle \mathbb{1}_{\left\{u:\left|k\left(X_{s}, u\right)\right| \geq 1\right\}}(z) \tilde{v}(\mathrm{~d} z) \mathrm{d} s \\
& +\int_{0}^{t} \int_{\mathbb{R}}\left(f_{n}\left(X_{s-}+k\left(X_{s-}, z\right)\right)-f_{n}\left(X_{s-}\right)\right)(\tilde{\mu}(\cdot, \mathrm{d} z, \mathrm{~d} s)-\tilde{v}(\mathrm{~d} z) \mathrm{d} s) \\
& +\int_{0}^{t} \int_{\mathbb{R}}\left(f_{n}\left(X_{s}+k\left(X_{s}, z\right)\right)-f_{n}\left(X_{s}\right)-\left\langle\nabla f_{n}\left(X_{s}\right), k\left(X_{s}, z\right)\right\rangle\right) \tilde{v}(\mathrm{~d} z) \mathrm{d} s \\
= & f_{n}\left(X_{0}\right)+\int_{0}^{t}\left\langle\nabla f_{n}\left(X_{s}\right), b^{*}\left(X_{s}\right)\right\rangle \mathrm{d} s \\
& +\int_{0}^{t} \int_{\mathbb{R}}\left(f_{n}\left(X_{s-}+k\left(X_{s-}, z\right)\right)-f_{n}\left(X_{s-}\right)\right)(\tilde{\mu}(\cdot, \mathrm{d} z, \mathrm{~d} s)-\tilde{v}(\mathrm{~d} z) \mathrm{d} s)
\end{aligned}
$$




$$
+\int_{0}^{t} \int_{\mathbb{R}^{d}}\left(f_{n}\left(X_{s}+k\left(X_{s}, z\right)\right)-f_{n}\left(X_{S}\right)-\left\langle\nabla f_{n}\left(X_{S}\right), k\left(X_{s}, z\right)\right\rangle\right) \tilde{v}(\mathrm{~d} z) \mathrm{d} s .
$$

Now, by letting $n \rightarrow \infty$ we see that the left hand-side converges to $f\left(X_{t}\right)$, and the first two terms on the right-hand side (for the second term we employ the dominated convergence theorem) converge to $f\left(X_{0}\right)$ and $\int_{0}^{t}\left\langle\nabla f\left(X_{s-}\right), b^{*}\left(X_{s-}\right)\right\rangle \mathrm{d} s$, respectively. Further, Taylor's theorem together with the fact that $m_{\phi}>1$ implies

$$
\begin{aligned}
& \left|f_{n}(x+y)-f_{n}(x)-\left\langle\nabla f_{n}(x), y\right\rangle\right| \\
& \leq \int_{0}^{1}\left|\nabla f_{n}(x+r y)-\nabla f_{n}(x)\right||y| \mathrm{d} r \\
& \leq\left\|f_{n}\right\|_{\phi} \phi(|y|) \mathbb{1}_{B_{1}(0)}(y)+2\left\|f_{n}\right\|_{\phi}|y| \mathbb{1}_{B_{1}^{c}(0)}(y) \\
& \leq\|f\|_{\phi} \phi(|y|) \mathbb{1}_{B_{1}(0)}(y)+2\|f\|_{\phi}|y| \mathbb{1}_{B_{1}^{c}(0)}(y) .
\end{aligned}
$$

Observe that according to Eq. (2.1) and (C3),

$$
\sup _{x \in \mathbb{R}^{d}} \int_{\mathbb{R}^{d}}\left(\phi(|y|) \mathbb{1}_{B_{1}(0)}(y)+|y| \mathbb{1}_{B_{1}^{c}(0)}(y)\right) v(x, \mathrm{~d} y)<\infty .
$$

Thus, the dominated convergence theorem implies that the last term converges to

$$
\int_{0}^{t} \int_{\mathbb{R}^{d}}\left(f\left(X_{s}+k\left(X_{s}, z\right)\right)-f\left(X_{s}\right)-\left\langle\nabla f\left(X_{s}\right), k\left(X_{s}, z\right)\right\rangle\right) \tilde{v}(\mathrm{~d} z) \mathrm{d} s .
$$

Finally, by employing the isometry formula, we have

$$
\begin{aligned}
\widetilde{\mathbb{E}}_{x} & {\left[\left(\int_{0}^{t} \int_{\mathbb{R}}\left(f_{n}\left(X_{S-}+k\left(X_{S-}, z\right)\right)-f_{n}\left(X_{S-}\right)-f\left(X_{S-}+k\left(X_{S-}, z\right)\right)+f\left(X_{S-}\right)\right)\right.\right.} \\
& \left.(\tilde{\mu}(\cdot, \mathrm{d} z, \mathrm{~d} s)-\tilde{v}(\mathrm{~d} z) \mathrm{d} s))^{2}\right] \\
& =\widetilde{\mathbb{E}}_{x}\left[\int_{0}^{t} \int_{\mathbb{R}}\left(f_{n}\left(X_{S}+k\left(X_{S}, z\right)\right)-f_{n}\left(X_{S}\right)-f\left(X_{S-}+k\left(X_{S}, z\right)\right)+f\left(X_{S}\right)\right)^{2} \tilde{v}(\mathrm{~d} z) \mathrm{d} s\right] .
\end{aligned}
$$

Now, since

$$
\begin{aligned}
& \left|f_{n}(x+y)-f_{n}(x)-f(x+y)+f(x)\right|^{2} \\
& \leq\left(\int_{0}^{1}\left(\left|\nabla f_{n}(x+r y)\right|+|\nabla f(x+r y)|\right)|y| \mathrm{d} r\right)^{2} \\
& \leq 4\|f\|_{1}^{2}|y|^{2},
\end{aligned}
$$

the dominated convergence theorem implies that, possibly passing to a subsequence, the third term on the left-hand side in Eq. (2.2) converges to

$$
\int_{0}^{t} \int_{\mathbb{R}^{d}}\left(f\left(X_{s-}+k\left(X_{s-}, z\right)\right)-f\left(X_{s-}\right)\right)(\tilde{\mu}(\cdot, \mathrm{d} z, \mathrm{~d} s)-\tilde{v}(\mathrm{~d} z) \mathrm{d} s),
$$

which proves the desired result.

Finally, by following the above arguments, one easily sees that under Eq. (1.14) the assertion also holds for any $f \in C_{b}^{1}\left(\mathbb{R}^{d}\right)$, which concludes the proof. 
Proof of Theorem 1.4 (b). We now prove the main result of this article. We follow the approach from [31]. Let $\beta \in C_{b}^{\varphi \psi}\left(\mathbb{R}^{d}\right)$ be a $\tau$-periodic solution to Eq. (1.12) discussed above. Recall that either $\beta \in C_{b}^{2}\left(\mathbb{R}^{d}\right)$ if $c(x) \not \equiv 0$, or $m_{\varphi \psi}>1$ [resp. $\beta \in C_{b}^{1}\left(\mathbb{R}^{d}\right)$ ] if $c(x) \equiv 0$ and Eq. (1.13) [resp. Eq. (1.14)] holds true, as assumed in Theorem 1.4 (b) (1), (2) and (3). According to Proposition 2.2, we can apply Itô's formula to the process $\left\{\beta\left(X_{t}\right)\right\}_{t \geq 0}$. Let us consider now the process $\left\{X_{t}-\overline{b^{*}} t-\beta\left(X_{t}\right)+\beta\left(X_{0}\right)\right\}_{t \geq 0}$. By combining Eq. (1.10) and Itô's formula we have that

$$
\begin{aligned}
X_{t} & -\overline{b^{*}} t-\beta\left(X_{t}\right)+\beta\left(X_{0}\right) \\
= & x+\int_{0}^{t}\left(b\left(X_{s}\right)+\int_{B_{1}^{c}(0)} y v\left(X_{s}, \mathrm{~d} y\right)-\overline{b^{*}}\right) \mathrm{d} s+\int_{0}^{t} \tilde{\sigma}\left(X_{s}\right) \mathrm{d} \tilde{W}_{s} \\
& +\int_{0}^{t} \int_{\mathbb{R}} k\left(X_{s-}, z\right)(\tilde{\mu}(\cdot, \mathrm{d} z, \mathrm{~d} s)-\tilde{v}(\mathrm{~d} z) \mathrm{d} s) \\
& -\int_{0}^{t}\left\langle\nabla \beta\left(X_{s}\right), b\left(X_{s}\right)\right\rangle \mathrm{d} s-\int_{0}^{t}\left\langle\nabla \beta\left(X_{s}\right), \tilde{\sigma}\left(X_{s}\right) \mathrm{d} \tilde{W}_{s}\right\rangle \\
& -\frac{1}{2} \sum_{i, j=1}^{d} \int_{0}^{t} c_{i j}\left(X_{s}\right) \partial_{i j} \beta\left(X_{s}\right) \mathrm{d} s \\
& -\int_{0}^{t} \int_{\mathbb{R}}\left(\beta\left(X_{s-}+k\left(X_{s-}, z\right)\right)-\beta\left(X_{s-}\right)\right)(\tilde{\mu}(\cdot, \mathrm{d} z, \mathrm{~d} s)-\tilde{v}(\mathrm{~d} z) \mathrm{d} s) \\
& -\int_{0}^{t} \int_{\mathbb{R}}\left(\beta\left(X_{s}+k\left(X_{s}, z\right)\right)-\beta\left(X_{s}\right)\right. \\
& \left.-\left\langle\nabla \beta\left(X_{s}\right), k\left(X_{s}, z\right)\right\rangle \mathbb{1}_{\left\{u:\left|k\left(X_{s}, u\right)\right|<1\right\}}(z)\right) \tilde{v}(\mathrm{~d} z) \mathrm{d} s \\
= & x+\int_{0}^{t} \tilde{\sigma}\left(X_{s}\right) \mathrm{d} \tilde{W}_{s}-\int_{0}^{t}\left\langle\nabla \beta\left(X_{s}\right), \tilde{\sigma}\left(X_{s}\right) \mathrm{d} \tilde{W}_{s}\right\rangle \\
& +\int_{0}^{t} \int_{\mathbb{R}}^{t} k\left(X_{s-}, z\right)(\tilde{\mu}(\cdot, \mathrm{d} z, \mathrm{~d} s)-\tilde{v}(\mathrm{~d} z) \mathrm{d} s) \\
- & \int_{0}^{t} \int_{\mathbb{R}}\left(\beta\left(X_{s-}+k\left(X_{s-}, z\right)\right)-\beta\left(X_{s-}\right)\right)(\tilde{\mu}(\cdot, \mathrm{d} z, \mathrm{~d} s)-\tilde{v}(\mathrm{~d} z) \mathrm{d} s),
\end{aligned}
$$

where we used the fact that $b^{*}\left(X_{t}\right)-\overline{b^{*}}=\mathcal{A}^{b} \beta\left(X_{t}\right)$ for any $t \geq 0$.

Clearly, $\left\{X_{t}-\overline{b^{*} t}-\beta\left(X_{t}\right)+\beta\left(X_{0}\right)\right\}_{t \geq 0}$ is a special semimartingale, and from [43, Proposition II.2.29] we see again that for the truncation function we can take $h(x)=x$. Thus, the first characteristic of $\left\{X_{t}-\overline{b^{*}} t-\beta\left(X_{t}\right)+\beta\left(X_{0}\right)\right\}_{t \geq 0}$ vanishes (that is, $B_{t} \equiv 0, t \geq 0$ ), the third characteristic equals to

$$
\begin{aligned}
C_{t}^{i j}= & \int_{0}^{t}\left(c_{i j}\left(X_{S}\right)+\sum_{k, l=1}^{d} c_{k l}\left(X_{S}\right) \partial_{k} \beta_{i}\left(X_{s}\right) \partial_{l} \beta_{j}\left(X_{S}\right)\right. \\
& \left.-\sum_{l=1}^{d} c_{i l}\left(X_{S}\right) \partial_{l} \beta_{j}\left(X_{S}\right)-\sum_{k=1}^{d} c_{k j}\left(X_{S}\right) \partial_{k} \beta_{i}\left(X_{S}\right)\right) \mathrm{d} s
\end{aligned}
$$




$$
=\int_{0}^{t} \sum_{k, l=1}^{d}\left(\delta_{k i}-\partial_{k} \beta_{i}\left(X_{s}\right)\right) c_{k l}\left(X_{s}\right)\left(\delta_{l j}-\partial_{l} \beta_{j}\left(X_{s}\right)\right) \mathrm{d} s,
$$

for $t \geq 0$ and $i, j=1, \ldots, d$, and the third modified characteristic reads

$$
\begin{aligned}
\widetilde{C}_{t}^{i j}= & C_{t}^{i j}+\int_{0}^{t} \int_{\mathbb{R}^{d}} y_{i} y_{j} v\left(X_{s}, \mathrm{~d} y\right) \mathrm{d} s \\
& +\int_{0}^{t} \int_{\mathbb{R}^{d}}\left(\left(\beta_{i}\left(X_{s}+y\right)-\beta_{i}\left(X_{s}\right)\right)\left(\beta_{j}\left(X_{s}+y\right)-\beta_{j}\left(X_{s}\right)\right)\right) v\left(X_{s}, \mathrm{~d} y\right) \mathrm{d} s \\
& -2 \int_{0}^{t} \int_{\mathbb{R}^{d}} y_{i}\left(\beta_{j}\left(X_{s}+y\right)-\beta_{j}\left(X_{s}\right)\right) v\left(X_{s}, \mathrm{~d} y\right) \mathrm{d} s,
\end{aligned}
$$

for $t \geq 0$ and $i, j=1, \ldots, d$. Also, from [43, Proposition II.2.17] and [70, Theorem 3.5] we see that the second characteristic is

$$
N(B, \mathrm{~d} s)=\int_{\mathbb{R}^{d}} \mathbb{1}_{B}\left(y-\left(\beta\left(X_{s}+y\right)-\beta\left(X_{s}\right)\right)\right) v\left(X_{s}, \mathrm{~d} y\right) \mathrm{d} s, \quad B \in \mathcal{B}\left(\mathbb{R}^{d}\right) .
$$

Consequently, for any $x \in \mathbb{R}^{d}$,

$$
\left\{\varepsilon X_{\varepsilon^{-2} t}-\bar{b}^{*} \varepsilon^{-1} t-\varepsilon \beta\left(X_{\varepsilon^{-2} t}\right)+\varepsilon \beta\left(X_{0}\right)\right\}_{t \geq 0}, \quad \varepsilon>0,
$$

is a $\mathbb{P}_{x}$ - semimartingale (with respect to the natural filtration generated by $\left\{X_{t}\right\}_{t \geq 0}$ ) whose (modified) characteristics (relative to $h(x)=x$ ) are given by

$$
\begin{aligned}
B_{t}^{\varepsilon, i}= & 0 \\
C_{t}^{\varepsilon, i j}= & \varepsilon^{2} \int_{0}^{\varepsilon^{-2} t} \sum_{k, l=1}^{d}\left(\delta_{k i}-\partial_{k} \beta_{i}\left(X_{s}\right)\right) c_{k l}\left(X_{s}\right)\left(\delta_{l j}-\partial_{l} \beta_{j}\left(X_{s}\right)\right) \mathrm{d} s, \\
N^{\varepsilon}(\mathrm{d} s, B)= & \frac{1}{\varepsilon^{2}} \int_{\mathbb{R}^{d}} \mathbb{1}_{B}\left(\varepsilon y-\varepsilon\left(\beta\left(X_{\varepsilon^{-2} s}+y\right)-\beta\left(X_{\varepsilon^{-2} s}\right)\right)\right) v\left(X_{\varepsilon^{-2} s}, \mathrm{~d} y\right) \mathrm{d} s, \\
\widetilde{C}_{t}^{\varepsilon, i j}= & C_{t}^{\varepsilon, i j}+\varepsilon^{2} \int_{0}^{\varepsilon^{-2} t} \int_{\mathbb{R}^{d}} y_{i} y_{j} v\left(X_{s}, \mathrm{~d} y\right) \mathrm{d} s \\
& +\varepsilon^{2} \int_{0}^{\varepsilon^{-2} t} \int_{\mathbb{R}^{d}}\left(\beta_{i}\left(X_{s}+y\right)-\beta_{i}\left(X_{s}\right)\right) \\
& \left.-2 \varepsilon^{2} \int_{0}^{\varepsilon^{-2} t} \int_{\mathbb{R}^{d}} y_{i}\left(X_{j}+y\right)-\beta_{j}\left(X_{s}\right)\right) v\left(X_{s}, \mathrm{~d} y\right) \mathrm{d} s
\end{aligned}
$$

$t \geq 0, B \in \mathcal{B}\left(\mathbb{R}^{d}\right), i, j=1, \ldots, d$, (see [70, Lemma 3.2 and Theorem 3.5] and [43, Proposition II.2.17]).

Further, observe that due to boundedness of $\beta(x),\left\{\varepsilon X_{\varepsilon^{-2} t}-\bar{b}^{*} \varepsilon^{-1} t-\varepsilon \beta\left(X_{\varepsilon^{-2} t}\right)+\right.$ $\left.\varepsilon \beta\left(X_{0}\right)\right\}_{t \geq 0}$ converges in the Skorohod space as $\varepsilon \rightarrow 0$ if, and only if, $\left\{\varepsilon X_{\varepsilon^{-2} t}-\right.$ $\left.\bar{b}^{*} \varepsilon^{-1} t\right\}_{t \geq 0}$ converges, and if this is the case the limit is the same. Now, according to 
[43, Theorem VIII.2.17], in order to prove the desired convergence it suffices to prove that

$$
\sup _{0 \leq s \leq t} B_{s}^{\varepsilon, i} \underset{\varepsilon \rightarrow 0}{\stackrel{\mathbb{P}_{x}}{\longrightarrow}} 0,
$$

for all $t \geq 0$ and $i=1, \ldots, d$, which is trivially satisfied,

$$
\int_{0}^{t} \int_{\mathbb{R}^{d}} g(y) N^{\varepsilon}(\mathrm{d} y, \mathrm{~d} s) \underset{\varepsilon \rightarrow 0}{\stackrel{\mathbb{P}_{x}}{\longrightarrow}} 0,
$$

for all $t \geq 0$ and $g \in C_{b}\left(\mathbb{R}^{d}\right)$ vanishing in a neighborhood of the origin, and

$$
\widetilde{C}_{t}^{\varepsilon, i j} \underset{\varepsilon \rightarrow 0}{\stackrel{\mathbb{P}_{x}}{\longrightarrow}} t \Sigma^{i j}
$$

for all $t \geq 0$ and $i, j=1, \ldots, d$, where $\Sigma$ is given in Eq. (1.3) and $\stackrel{\mathbb{P}_{x}}{\rightarrow}$ stands for the convergence in probability.

To prove the convergence in Eq. (2.4), first observe that due to $\tau$-periodicity of all components we can replace $\left\{X_{t}\right\}_{t \geq 0}$ by $\left\{\Pi\left(X_{t}\right)\right\}_{t \geq 0}$, which is an ergodic Markov process (see Proposition 1.1). The assertion now follows as a direct consequence of the Birkhoff ergodic theorem.

To prove the relation in Eq. (2.3) we proceed as follows. Fix $g \in C_{b}\left(\mathbb{R}^{d}\right)$ that vanishes on $B_{\delta}(0)$ for some $\delta>0$. Define now

$$
\begin{aligned}
F^{\varepsilon}(x):= & \frac{1}{\varepsilon^{2}} \int_{\mathbb{R}^{d}} g(\varepsilon y-\varepsilon(\beta(x+y)-\beta(x))) v(x, \mathrm{~d} y) \\
& -\frac{1}{\varepsilon^{2}} \int_{\mathbb{T}_{\tau}^{d}} \int_{\mathbb{R}^{d}} g(\varepsilon y-\varepsilon(\beta(z+y)-\beta(z))) v(z, \mathrm{~d} y) \pi(\mathrm{d} z) .
\end{aligned}
$$

Clearly, for any $\varepsilon>0, F^{\varepsilon}(x)$ is bounded, $\tau$-periodic, and satisfies $F^{\varepsilon}\left(X_{t}\right)=F^{\varepsilon}$ $\left(\Pi_{\tau}\left(X_{t}\right)\right)$ for $t \geq 0$, and

$$
\int_{\mathbb{T}_{\tau}^{d}} F^{\varepsilon}(x) \pi(\mathrm{d} x)=0 .
$$

Now, by the Markov property and exponential ergodicity of $\left\{\Pi_{\tau}\left(X_{t}\right)\right\}_{t \geq 0}$, we have

$$
\begin{aligned}
& \mathbb{E}_{x}\left[\left(\int_{0}^{t} F^{\varepsilon}\left(X_{\varepsilon^{-2} s}\right) \mathrm{d} s\right)^{2}\right] \\
& =\mathbb{E}_{x_{\tau}}^{\tau}\left[\left(\int_{0}^{t} F^{\varepsilon}\left(\Pi_{\tau}\left(X_{\varepsilon^{-2} s}\right)\right) \mathrm{d} s\right)^{2}\right] \\
& =2 \int_{0}^{t} \int_{0}^{s} \mathbb{E}_{x_{\tau}}^{\tau}\left[F^{\varepsilon}\left(\Pi_{\tau}\left(X_{\varepsilon^{-2} s}\right)\right) F^{\varepsilon}\left(\Pi_{\tau}\left(X_{\varepsilon^{-2} u}\right)\right)\right] \mathrm{d} u \mathrm{~d} s \\
& =2 \int_{0}^{t} \int_{0}^{s} \mathbb{E}_{x_{\tau}}^{\tau}\left[F^{\varepsilon}\left(\Pi_{\tau}\left(X_{\varepsilon^{-2} u}\right)\right) P_{\varepsilon^{-2}(s-u)}^{\tau} F^{\varepsilon}\left(\Pi_{\tau}\left(X_{\varepsilon^{-2} u}\right)\right)\right] \mathrm{d} u \mathrm{~d} s
\end{aligned}
$$




$$
\begin{aligned}
& \leq 2 \Gamma\left\|F^{\varepsilon}\right\|_{\infty}^{2} \int_{0}^{t} \int_{0}^{s} \mathrm{e}^{-\gamma \varepsilon^{-2}(s-u)} \mathrm{d} u \mathrm{~d} s \\
& \leq \frac{2 \Gamma \varepsilon^{2} t}{\gamma}\left\|F^{\varepsilon}\right\|_{\infty}^{2} \\
& \leq \frac{8 \Gamma\|g\|_{\infty}^{2} t}{\varepsilon^{2} \gamma} \sup _{x_{\tau} \in \mathbb{T}_{\tau}^{d}}\left|\int_{\mathbb{R}^{d}} \mathbb{1}_{B_{\delta}^{c}}(\varepsilon y-\varepsilon(\beta(x+y)-\beta(x))) v(x, \mathrm{~d} y)\right|^{2} .
\end{aligned}
$$

Let $\varepsilon>0$ be such that $2 \varepsilon\|\beta\|_{\infty}<\delta / 2$. Then,

$$
\begin{aligned}
\mathbb{E}_{x}\left[\left(\int_{0}^{t} F^{\varepsilon}\left(X_{\varepsilon^{-2} s}\right) \mathrm{d} s\right)^{2}\right] & \leq \frac{8 \Gamma\|g\|_{\infty}^{2} t}{\varepsilon^{2} \gamma} \sup _{x_{\tau} \in \mathbb{T}_{\tau}^{d}}\left|\int_{\mathbb{R}^{d}} \mathbb{1}_{B_{\delta / 2}^{c}}(\varepsilon y) v(x, \mathrm{~d} y)\right|^{2} \\
& =\frac{8 \Gamma\|g\|_{\infty}^{2} t}{\varepsilon^{2} \gamma} \sup _{x_{\tau} \in \mathbb{T}_{\tau}^{d}}\left|v\left(x, B_{\delta / 2 \varepsilon}^{c}\right)\right|^{2} .
\end{aligned}
$$

Now, since

$$
\frac{\delta^{2}}{4 \varepsilon^{2}} v\left(x, B_{\delta / 2 \varepsilon}^{c}\right) \leq \int_{B_{\delta / 2 \varepsilon}^{c}}|y|^{2} v(x, \mathrm{~d} y)
$$

we have that

$$
\mathbb{E}_{x}\left[\left(\int_{0}^{t} F^{\varepsilon}\left(X_{\varepsilon^{-2} s}\right) \mathrm{d} s\right)^{2}\right] \leq \frac{128 \Gamma\|g\|_{\infty}^{2} \varepsilon^{2} t}{\gamma \delta^{4}} \sup _{x_{\tau} \in \mathbb{T}_{\tau}^{d}}\left(\int_{B_{\delta / 2 \varepsilon}^{c}}|y|^{2} v(x, \mathrm{~d} y)\right)^{2} .
$$

Consequently,

$$
\begin{aligned}
& \left(\mathbb{E}_{x}\left[\left(\int_{0}^{t} \int_{\mathbb{R}^{d}} g(y) N^{\varepsilon}(\mathrm{d} y, \mathrm{~d} s)\right)^{2}\right]\right)^{\frac{1}{2}} \\
& \leq\left(\mathbb{E}_{x}\left[\left(\int_{0}^{t} F^{\varepsilon}\left(X_{\varepsilon^{-2} s}\right) \mathrm{d} s\right)^{2}\right]\right)^{\frac{1}{2}} \\
& +\left(\mathbb{E}_{x}\left[\left(\frac{t}{\varepsilon^{2}} \int_{\mathbb{T}_{\tau}^{d}} \int_{\mathbb{R}^{d}} g(\varepsilon y-\varepsilon(\beta(z+y)-\beta(z))) v(z, \mathrm{~d} y) \pi(\mathrm{d} z)\right)^{2}\right]\right)^{\frac{1}{2}} \\
& \leq \frac{8 \sqrt{2} \Gamma^{1 / 2}\|g\|_{\infty} \varepsilon t^{1 / 2}}{\gamma^{1 / 2} \delta^{2}} \sup _{x_{\tau} \in \mathbb{T}_{\tau}^{d}} \int_{B_{\delta / 2 \varepsilon}^{c}}|y|^{2} v(x, \mathrm{~d} y) \\
& +\frac{4\|g\|_{\infty} t}{\delta^{2}} \int_{\mathbb{T}_{\tau}^{d}} \int_{B_{\delta / 2 \varepsilon}^{c}} \frac{\delta^{2}}{4 \varepsilon^{2}} v(z, \mathrm{~d} y) \pi(\mathrm{d} z) \\
& \leq\left(\frac{8 \sqrt{2} \Gamma^{1 / 2}\|g\|_{\infty} \varepsilon t^{1 / 2}}{\gamma^{1 / 2} \delta^{2}}+\frac{4\|g\|_{\infty} t}{\delta^{2}}\right)_{x_{\tau} \in \mathbb{T}_{\tau}^{d}} \int_{B_{\delta / 2 \varepsilon}^{c}}|y|^{2} v(x, \mathrm{~d} y),
\end{aligned}
$$

which together with $(\mathbf{C 3})$ concludes the proof. 


\section{On structural properties of LTPs}

In this section, we present sufficient conditions for LTPs satisfying strong Feller property, open-set irreducibility, regularity property of the semigroup, and regularity properties of the solution to the Poisson equation Eq. (1.11), respectively. Several examples are also included.

Strong Feller property. Let $\left\{X_{t}\right\}_{t \geq 0}$ be a Lévy-type process with symbol $q(x, \xi)$ and Lévy triplet $(b(x), c(x), v(x, \mathrm{~d} y))$.

(i) Let $\left\{X_{t}\right\}_{t \geq 0}$ be a diffusion process (that is, $\left.v(x, \mathrm{~d} y) \equiv 0\right)$. According to [66, Theorem V.24.1], it will be strong Feller if $b(x)$ is measurable, $c(x)$ is continuous and positive definite, and there is a constant $\Lambda>0$ such that

$$
\left|c_{i j}(x)\right|+\left|b_{i}(x)\right|^{2} \leq \Lambda\left(1+|x|^{2}\right), \quad x \in \mathbb{R}^{d}, i, j=1, \ldots, d .
$$

Let us also remark that when $\left\{X_{t}\right\}_{t \geq 0}$ is a diffusion process generated with a second-order elliptic operator in divergence form

$$
\mathcal{L} f(x)=\nabla(c(x) \cdot \nabla f(x))
$$

with $c(x)$ bounded, measurable and uniformly elliptic, strong Feller property of $\left\{X_{t}\right\}_{t \geq 0}$ has been discussed in $[5,59,80]$.

(ii) Suppose that $(x, \xi) \mapsto q(x, \xi)$ is continuous, $b(x)$ is continuous and bounded, $c(x)$ is continuous, bounded and positive definite, and $x \mapsto \int_{B}\left(1 \wedge|y|^{2}\right) v(x, \mathrm{~d} y)$ is continuous and bounded for any $B \in \mathcal{B}\left(\mathbb{R}^{d}\right)$. Then, according to [14, Theorems 3.23, 3.24 and 3.25] and [79, Theorem 4.3 and its remark], $\left\{X_{t}\right\}_{t \geq 0}$ is strong Feller.

(iii) Recently, there are lots of developments on heat kernel (that is, the transition density function) estimates of Feller processes. The reader is referred to [17$20,36,46,47]$ and the references therein for more details. In particular, let

$\mathcal{L} f(x)=\int_{\mathbb{R}^{d}}\left(f(x+y)-f(x)-\langle\nabla f(x), y\rangle \mathbb{1}_{B_{1}(0)}(y)\right) \frac{\kappa(x, y)}{|y|^{d+\alpha(x)}} \mathrm{d} y$,

where $\alpha: \mathbb{R}^{d} \rightarrow(0,2)$ is a Hölder continuous function such that

$$
\begin{aligned}
& 0<\alpha_{1} \leq \alpha(x) \leq \alpha_{2}<2, \quad x \in \mathbb{R}^{d}, \\
& |\alpha(x)-\alpha(y)| \leq c_{1}\left(|x-y|^{\beta_{1}} \wedge 1\right), \quad x, y \in \mathbb{R}^{d},
\end{aligned}
$$

for some constants $c_{1}>0$ and $\beta_{1} \in(0,1]$, and $\kappa: \mathbb{R}^{d} \times \mathbb{R}^{d} \rightarrow(0, \infty)$ is a measurable function satisfying

$$
\begin{aligned}
& \kappa(x, y)=\kappa(x,-y), \quad x, y \in \mathbb{R}^{d}, \\
& 0<\kappa_{1} \leq \kappa(x, y) \leq \kappa_{2}<\infty, \quad x, y \in \mathbb{R}^{d}, \\
& |\kappa(x, y)-\kappa(\bar{x}, y)| \leq c_{2}\left(|x-\bar{x}|^{\beta_{2}} \wedge 1\right), \quad x, \bar{x}, y \in \mathbb{R}^{d},
\end{aligned}
$$


for some constants $c_{2}>0$ and $\beta_{2} \in(0,1]$. If $\left(\alpha_{2} / \alpha_{1}\right)-1<\bar{\beta}_{0} / \alpha_{2}$, with $\bar{\beta}_{0} \in\left(0, \beta_{0}\right] \cap\left(0, \alpha_{2} / 2\right)$ and $\beta_{0}=\min \left\{\beta_{1}, \beta_{2}\right\}$, then, by [17, Thereoms 1.1 and 1.3], $\left(\mathcal{L}, C_{c}^{\infty}\left(\mathbb{R}^{d}\right)\right)$ generates a LTP. Furthermore, by upper bounds as well as Hölder regularity and gradient estimates of the heat kernel (see [17, Thereoms 1.1 and 1.3, and Remark 1.4]), this associated process is strong Feller.

(iv) Let $\left\{X_{t}\right\}_{t \geq 0}$ and $\left\{\tilde{X}_{t}\right\}_{t \geq 0}$ be LTPs with semigroups $\left\{P_{t}\right\}_{t \geq 0}$ and $\left\{\tilde{P}_{t}\right\}_{t \geq 0}$, and Feller generators $\left(\mathcal{A}^{\infty}, \mathcal{D}_{\mathcal{A}^{\infty}}\right)$ and $\left(\tilde{\mathcal{A}}^{\infty}, \mathcal{D}_{\tilde{\mathcal{A}}^{\infty}}\right)$, respectively. Suppose that $\left\{X_{t}\right\}_{t \geq 0}$ is strong Feller. If $\mathcal{A}^{\infty}-\tilde{\mathcal{A}}^{\infty}$ is a bounded operator on $\left(B_{b}\left(\mathbb{R}^{d}\right),\|\cdot\|_{\infty}\right)$, then the formula

$$
P_{t} f=\tilde{P}_{t} f+\int_{0}^{t} P_{s}\left(\mathcal{A}^{\infty}-\tilde{\mathcal{A}}^{\infty}\right) \tilde{P}_{t-s} f \mathrm{~d} s, \quad f \in \mathcal{D}_{\mathcal{A}^{\infty}} \cap \mathcal{D}_{\tilde{\mathcal{A}}^{\infty}},
$$

implies that $\left\{\tilde{X}_{t}\right\}_{t \geq 0}$ is also strong Feller. Namely, since both $\left\{X_{t}\right\}_{t \geq 0}$ and $\left\{\tilde{X}_{t}\right\}_{t \geq 0}$ are LTPs, the above relation holds for any $f \in C_{c}^{\infty}\left(\mathbb{R}^{d}\right)$. According to [21, Lemma 1.1.1], the boundedness of $\mathcal{A}^{\infty}-\tilde{\mathcal{A}}^{\infty}$ and the dominated convergence theorem, it also holds for $f(x)=\mathbb{1}_{O}(x)$ for any open set $O \subseteq \mathbb{R}^{d}$. The claim now follows from Dynkin's monotone class theorem. The assertion above roughly asserts that a bounded perturbation preserves the strong Feller property. Below is an example.

Let

$$
\begin{aligned}
\tilde{\mathcal{L}} f(x)= & \int_{B_{1}(0)}(f(x+y)-f(x)-\langle\nabla f(x), y\rangle) \frac{\kappa(x, y)}{|y|^{d+\alpha(x)}} \mathrm{d} y \\
& +\int_{B_{1}^{c}(0)}(f(x+y)-f(x)) v(x, \mathrm{~d} y),
\end{aligned}
$$

where $\alpha(x)$ and $\kappa(x, y)$ satisfy all the assumptions in (iii), and $v(x, \mathrm{~d} y)$ is such that

(a) $\sup v\left(x, B_{1}(0)\right)=0$;

$x \in \mathbb{R}^{d}$

(b) $\sup _{x \in \mathbb{R}^{d}} \int_{\mathbb{R}^{d}}|y|^{2} v(x, \mathrm{~d} y)<\infty$;

(c) $f \mapsto \int_{\mathbb{R}^{d}}(f(\cdot+y)-f(\cdot)) v(\cdot, \mathrm{d} y)$ is an operator on $C_{\infty}\left(\mathbb{R}^{d}\right)$.

For example, one can take $v(x, \mathrm{~d} y)=\frac{\gamma(x, y)}{|y|^{d+\delta}} \mathbb{1}_{B_{1}^{c}(0)}(y) \mathrm{d} y$ with $\delta>0$ and $\gamma(x, y)$ nonnegative, bounded and such that $x \mapsto \gamma(x, y)$ is continuous for almost every $y \in \mathbb{R}^{d}$. Further, let $\mathcal{L}$ be the operator given in (iii). Then,

$$
\begin{aligned}
(\tilde{\mathcal{L}}-\mathcal{L}) f(x)= & \int_{B_{1}^{c}(0)}(f(x+y)-f(x)) v(x, \mathrm{~d} y) \\
& -\int_{B_{1}^{c}(0)}(f(x+y)-f(x)) \frac{\kappa(x, y)}{|y|^{d+\alpha(x)}} \mathrm{d} y
\end{aligned}
$$

is bounded on $\left(B_{b}\left(\mathbb{R}^{d}\right),\|\cdot\|_{\infty}\right)$. By assumption, it is also bounded on $\left(C_{\infty}\left(\mathbb{R}^{d}\right)\right.$, $\left.\|\cdot\|_{\infty}\right)$. Now, according to [14, Lemma 1.28] and [77, Proposition 2.1], $\tilde{\mathcal{L}}=$ 
$\mathcal{L}+(\tilde{\mathcal{L}}-\mathcal{L})$ generates a LTP. This, along with the assertion above and the strong Feller property of the process associated with $\mathcal{L}$, yields the strong Feller property of the process associated with $\tilde{\mathcal{L}}$.

We remark also that the strong Feller property of LTPs has been discussed in [74]. In the special case when $\left\{X_{t}\right\}_{t \geq 0}$ is given through Eq. (1.7), the strong Feller property (and the open-set irreducibility) has been discussed in [50] under the assumption that $v_{Z}\left(\mathbb{R}^{m}\right)<\infty$, and in $[55,56]$ for an arbitrary $v_{Z}(\mathrm{~d} y)$, that is, an arbitrary pure-jump Lévy process $\left\{Z_{t}\right\}_{t \geq 0}$. Observe that in both situations non-degeneracy of $\Phi_{2}(x) \Phi_{2}^{\prime}(x)$ has been assumed. In the case when $\Phi_{3}(x) \equiv \Phi_{3} \in \mathbb{R}^{d \times q}$ the problem has been considered in $[3,51,54]$, and for non-constant (and non-degenerated) $\Phi_{3}(x)$ in [52].

Open-set irreducibility. Let $\left\{X_{t}\right\}_{t \geq 0}$ be a Lévy-type process with symbol $q(x, \xi)$ and Lévy triplet $(b(x), c(x), v(x, \mathrm{~d} y))$.

(i) According to [66, Theorems V.20.1 and V.24.1] and [26, Theorem 7.3.8], a diffusion process will be open-set irreducible (and strong Feller) if $b(x)$ and $c(x)$ are locally Hölder continuous, $c(x)$ is positive definite, and Eq. (3.1) holds true. Observe that Eq. (3.1) trivially holds true in the periodic case.

Also, when $b(x) \in C_{b}^{1}\left(\mathbb{R}^{d}\right), c(x) \in C_{b}^{2}\left(\mathbb{R}^{d}\right), \partial_{i j} c_{k l}(x)$ is uniformly continuous for all $i, j, k, l=1, \ldots, d$, and $c(x)$ is positive definite, open-set irreducibility (and strong Feller property) of the process follows from the support theorem for diffusion processes, see [34, Lemma 6.1.1] and [40, p. 517]. For support theorem of jump processes one can refer to [78].

(ii) If $\left\{X_{t}\right\}_{t \geq 0}$ is a diffusion process generated by a second-order elliptic operator in divergence form Eq. (3.2) with uniformly elliptic, bounded and measurable diffusion coefficient, open-set irreducibility (and strong Feller property) follows from the corresponding heat kernel estimates (see [5,59,80]).

The diffusion processes with jumps or pure jump process considered in [17$20,36,46,47]$ are also open-set irreducible, which is a direct consequence of obtained lower bounds of heat kernel.

(iii) Let $\mathcal{L}$ and $\tilde{\mathcal{L}}$ be the operators from (iv) in the discussion on the strong Feller property. According to [17, Thereom 1.3], the LTP corresponding to $\mathcal{L}$ is openset irreducible. Further, observe that

$$
\sup _{x \in \mathbb{R}^{d}} \int_{B_{1}^{c}(0)} \frac{\kappa(x, y)}{|y|^{d+\alpha(x)}} \mathrm{d} y<\infty .
$$

Thus, by [9, Lemma 3.1] and [8, Lemma 3.6], the process associated with the operator $\tilde{\mathcal{L}}$ is also open-set irreducible.

For open-set irreducibility of LTPs of the form Eq. (1.6) we refer the reader to [3,50] and $[55,56]$.

In the following proposition, which slightly generalizes [37, Lemma 2], we show that a LTP will be open-set irreducible if the corresponding Lévy measure shows enough jump activity. First, recall that a function $f: \mathbb{R}^{d} \rightarrow \mathbb{R}$ is said to be lower 
semi-continuous if

$$
\liminf _{y \rightarrow x} f(y) \geq f(x), \quad x \in \mathbb{R}^{d} .
$$

Proposition 3.1. The process $\left\{X_{t}\right\}_{t \geq 0}$ will be open-set irreducible if there are constants $R>r \geq 0$ such that

(i) $\inf _{x \in K} v(x, O)>0$ for every non-empty open set $O \subseteq B_{R}(0) \backslash B_{r}(0)$, and every non-empty compact set $K \subset \mathbb{R}^{d}$;

(ii) the function $x \mapsto \int_{\mathbb{R}^{d}} f(y+x) v(x, \mathrm{~d} y)$ is lower semi-continuous for every non-negative lower semi-continuous function $f: \mathbb{R}^{d} \rightarrow \mathbb{R}$.

Proof. Let $x \in \mathbb{R}^{d}$ and $\rho>0$ be arbitrary, and let $f \in C_{c}^{\infty}\left(\mathbb{R}^{d}\right)$ be such that $0 \leq f \leq 1$ and supp $f \subset B_{\rho}(x)$. By assumption,

$$
\lim _{t \rightarrow 0}\left\|\frac{P_{t} f-f}{t}-\mathcal{A}^{\infty} f\right\|_{\infty}=0 .
$$

In particular, for any $B \subseteq B_{\rho}^{c}(x)$,

$$
\begin{aligned}
\liminf _{t \rightarrow 0} \inf _{y \in B} \frac{\mathbb{P}_{y}\left(X_{t} \in B_{\rho}(x)\right)}{t} & \geq \liminf _{t \rightarrow 0} \inf _{y \in B} \frac{P_{t} f(y)}{t} \\
& =\liminf _{t \rightarrow 0} \inf _{y \in B}\left|\frac{P_{t} f(y)}{t}-\mathcal{A}^{\infty} f(y)+\mathcal{A}^{\infty} f(y)\right| \\
& =\inf _{y \in B}\left|\mathcal{A}^{\infty} f(y)\right| \\
& =\inf _{y \in B} \int_{\mathbb{R}^{d}} f(z+y) v(y, \mathrm{~d} z) .
\end{aligned}
$$

Further, let $0<\varepsilon<\rho$ be arbitrary, and let $0 \leq f_{\varepsilon} \in C_{c}^{\infty}\left(\mathbb{R}^{d}\right)$ be such that

$$
f_{\varepsilon}(y)=\left\{\begin{array}{l}
1, y \in B_{\rho-\varepsilon}(x) \\
0, y \in B_{\rho}^{c}(x) .
\end{array}\right.
$$

Then, for any $y \in B_{\rho}^{c}(x)$ we have that

$$
\begin{aligned}
\mathcal{A}^{\infty} f_{\varepsilon}(y) & =\int_{\mathbb{R}^{d}} f_{\varepsilon}(z+y) v(y, \mathrm{~d} z) \\
& \geq v\left(y,\left(B_{R}(0) \backslash B_{r}(0)\right) \cap B_{\rho-\varepsilon}(x-y)\right) .
\end{aligned}
$$

Next, take $x, y \in \mathbb{R}^{d}$ such that $r<|x-y|<R$, and pick $\varepsilon, \rho>0$ such that $\varepsilon<\rho$ and $r+2 \rho<|x-y|<R-2 \rho$. Then, we have

$$
\begin{aligned}
\liminf _{t \rightarrow 0} \inf _{z \in B_{\rho}(x)} \frac{\mathbb{P}_{z}\left(X_{t} \in B_{\rho}(y)\right)}{t} & \geq \inf _{z \in B_{\rho}(x)} v\left(z,\left(B_{R}(0) \backslash B_{r}(0)\right) \cap B_{\rho-\varepsilon}(y-z)\right) \\
& =\inf _{z \in B_{\rho}(x)} v\left(z, B_{\rho-\varepsilon}(y-z)\right) .
\end{aligned}
$$


Assume now that inf $\operatorname{zeB}_{\rho}(x) v\left(z, B_{\rho-\varepsilon}(y-z)\right)=0$. Then, there is a sequence $\left\{z_{n}\right\}_{n \in \mathbb{N}}$ $\subset B_{\rho}(x)$ converging to $z_{0} \in \bar{B}_{\rho}(x)$ such that

$$
\liminf _{n \rightarrow \infty} v\left(z_{n}, B_{\rho-\varepsilon}\left(y-z_{n}\right)\right)=\liminf _{n \rightarrow \infty} \int_{\mathbb{R}^{d}} \mathbb{1}_{B_{\rho-\varepsilon}(y)}\left(u+z_{n}\right) v\left(z_{n}, \mathrm{~d} u\right)=0 .
$$

However, since $z \mapsto \mathbb{1}_{B_{\rho-\varepsilon}(y)}(z)$ is a lower semi-continuous function, we have that

$$
\liminf _{n \rightarrow \infty} \int_{\mathbb{R}^{d}} \mathbb{1}_{B_{\rho-\varepsilon}(y)}\left(u+z_{n}\right) v\left(z_{n}, \mathrm{~d} u\right)=v\left(z_{0}, B_{\rho-\varepsilon}\left(y-z_{0}\right)\right)>0,
$$

which is in contradiction with the above assumption. Hence, there is $t_{*}=t_{*}(x, y, \rho, \varepsilon)$ $>0$ such that

$$
\mathbb{P}_{z}\left(X_{t} \in B_{\rho}(y)\right)>0, \quad z \in B_{\rho}(x), t \in\left(0, t_{*}\right] .
$$

Fix now $\varepsilon, \rho>0$ such that $\varepsilon<\rho$ and $4 \rho<R-r$. From the previous discussion it follows that for any $x, y \in \mathbb{R}^{d}$ with $r+2 \rho<|x-y|<R-2 \rho$, there is $t_{* *}=$ $t_{\text {** }}(x, y, \rho, \varepsilon)>0$ such that

$$
\mathbb{P}_{z}\left(X_{t} \in B_{\rho}(y)\right)>0, \quad z \in B_{\rho}(x), t \in\left(0, t_{* *}\right]
$$

The assertion now follows by employing the Chapman-Kolmogorov equation.

Observe that in Proposition 3.1 we require that $v(x, \mathrm{~d} y)$ is not singular with respect to the $d$-dimensional Lebesgue measure. However, there are many interesting openset irreducible LTPs which do not meet this property. For example, let $\left\{X_{t}\right\}_{t \geq 0}$ be a solution to Eq. (1.6) with $n=d+1, \Phi(x)=\left(\bar{\Phi}(x), \mathbb{I}_{d}\right)$ and $Y_{t}=\left(t, B_{t}, Z_{t}\right)^{\prime}, t \geq 0$, where $\bar{\Phi}: \mathbb{R}^{d} \rightarrow \mathbb{R}^{d}, \mathbb{I}_{d}$ is the $d \times d$-identity matrix, $\left\{B_{t}\right\}_{t \geq 0}$ is a $d_{1}$-dimensional Brownian motion with $1 \leq d_{1}<d$, and $\left\{Z_{t}\right\}_{t \geq 0}$ is a $\left(d-d_{1}\right)$-dimensional rotationally invariant $\alpha$-stable Lévy process (independent of $\left.\left\{B_{t}\right\}_{t \geq 0}\right)$ with $\alpha \in(0,2)$. Clearly, in this case the Lévy measure is $\left(d-d_{1}\right)$-dimensional. Thus, Proposition 3.1 cannot be applied to the process $\left\{X_{t}\right\}_{t \geq 0}$. However, open-set irreducibility of $\left\{X_{t}\right\}_{t \geq 0}$ may be concluded by employing the time-changed idea as in [84]. Namely, the Girsanov transformation implies open-set irreducibility of a solution to Eq. (1.6) with $\Phi_{0}(x)$ similar to $\Phi(x)$ defined above and $\bar{Y}_{t}=\left(t, B_{t}, \bar{B}_{t}\right)^{\prime}, t \geq 0$, where $\left\{B_{t}\right\}_{t \geq 0}$ is also as above, and $\left\{\bar{B}_{t}\right\}_{t \geq 0}$ is a $\left(d-d_{1}\right)$-dimensional Brownian motion (independent of $\left\{B_{t}\right\}_{t \geq 0}$ ). With this at hand, and following the approach in [84] (the time-changed idea combined with approximation argument), we conclude open-set irreducibility of $\left\{X_{t}\right\}_{t \geq 0}$. An alternative approach is based on the Levi's method from PDE theory. Namely, since the transition function of the process $\left\{\left(B_{t}, Z_{t}\right)\right\}_{t \geq 0}$ enjoys the product form with Gaussian estimates and two-sided heat kernel estimates for rotationally invariant $\alpha$-stable processes, one may follow the argument from [20] to get two-sided heat kernel estimates for $\left\{X_{t}\right\}_{t \geq 0}$. When $\alpha \in(0,1)$ we may need to additionally assume that $\Phi(x)$ is Hölder continuous.

Let us also remark that open-set irreducibility (and strong Feller property) of a solution to Eq. (1.6) with $\Phi(x)=\left(\bar{\Phi}(x), \mathbb{I}_{d}\right)$ and $Y_{t}=\left(t, Z_{t}^{1}, \ldots, Z_{t}^{d}\right)^{\prime}, t \geq 0$, 
where $\bar{\Phi}: \mathbb{R}^{d} \rightarrow \mathbb{R}^{d}$, and $\left\{Z_{t}^{i}\right\}_{t \geq 0}, i=1, \ldots, d$, are mutually independent onedimensional symmetric $\alpha$-stable Lévy processes with $\alpha \in(1,2)$, has been deduced in [1, Theorem 3.1(iv)]. Note that in this case the Lévy measure again does not satisfy (i) in Proposition 3.1.

Regularity property of the semigroup, and regularity properties of the solution to Eq. (1.11). Let $\left\{X_{t}\right\}_{t \geq 0}$ be a Lévy-type process with $\tau$-periodic Lévy triplet $(b(x), c(x), v(x, \mathrm{~d} y))$.

(i) (Diffusion processes) Let $\varepsilon \in(0,1)$, and let $\left\{X_{t}\right\}_{t \geq 0}$ be a diffusion process with coefficients $b \in C_{b}^{\varepsilon}\left(\mathbb{R}^{d}\right), c \in C_{b}^{1+\varepsilon}\left(\mathbb{R}^{d}\right)$, and $c(x)$ being also positive definite. Then, (C4)(i) with arbitrary $t_{0}>0$ and $\psi(r)=r^{\varepsilon}$ follows from [60, the proof of Lemma 2.3]. Also, a straightforward adaptation of [60, Theorem 2.1], together with [41, Chapter 4.8] and [61, Proposition 4.2], implies (C4)(ii) with $\varphi(r)=r^{2}$. Then, the conclusion of Theorem 1.4 holds.

(ii) (Diffusion processes with jumps) Let $\varepsilon \in(0,1)$. Assume that $b(x)$ and $c(x)$ are as in (i), and that $v(x, \mathrm{~d} y)$ satisfies

(a) $\sup _{x \in \mathbb{R}^{d}} \int_{B_{1}(0)}|z|^{1+\varepsilon} v(x, \mathrm{~d} z)<\infty$;

(b) $\lim _{\epsilon \rightarrow 0} \sup _{x \in \mathbb{R}^{d}} \int_{B_{\epsilon}(0)}|z|^{1+\varepsilon} v(x, \mathrm{~d} z)=0$;

(c) $\lim _{R \rightarrow \infty} \sup _{x \in \mathbb{R}^{d}} \int_{B_{R}^{c}(0)}|z|^{1+\varepsilon} v(x, \mathrm{~d} z)=0$;

(d) $\sup _{x, y \in \mathbb{R}^{d}}|x-y|^{-\varepsilon} \int_{\mathbb{R}^{d}}\left(1 \wedge|z|^{1+\varepsilon}\right)|v(x, \mathrm{~d} z)-v(y, \mathrm{~d} z)|<\infty$.

Here, $|\mu(\mathrm{d} z)|$ stands for the total variation measure of a signed measure $\mu(\mathrm{d} z)$. Then, (C4)(ii) with $\varphi(r)=r^{2}$ follows again from [60, Theorem 2.1], together with [41, Chapter 4.8] and [61, Proposition 4.2].

Let us give sufficient conditions that $\left\{X_{t}\right\}_{t \geq 0}$ also satisfies (C4)(i). Denote by $\left\{P_{t}\right\}_{t \geq 0}$ the semigroup of $\left\{X_{t}\right\}_{t \geq 0}$, and let $\left\{\tilde{P}_{t}\right\}_{t \geq 0}$ be the semigroup of the diffusion process with coefficients $b(x)$ and $c(x)$. Also, denote by $\left(\mathcal{A}^{\infty}, \mathcal{D}_{\mathcal{A}}\right)$ and $\left(\tilde{\mathcal{A}}^{\infty}, \mathcal{D}_{\tilde{\mathcal{A}}^{\infty}}\right)$ the corresponding $C_{\infty}$-generators, respectively. Then,

$$
P_{t} f=\tilde{P}_{t} f+\int_{0}^{t} \tilde{P}_{s}\left(\mathcal{A}^{\infty}-\tilde{\mathcal{A}}^{\infty}\right) P_{t-s} f \mathrm{~d} s, \quad f \in \mathcal{D}_{\mathcal{A}^{\infty}} \cap \mathcal{D}_{\tilde{\mathcal{A}}^{\infty}} .
$$

Since both processes are LTPs, the above relation holds for any $f \in C_{c}^{\infty}\left(\mathbb{R}^{d}\right)$. Assume next that $\mathcal{A}^{\infty}-\tilde{\mathcal{A}}^{\infty}$ is a bounded operator on $\left(B_{b}\left(\mathbb{R}^{d}\right),\|\cdot\|_{\infty}\right)$. Then, according to [21, Lemma 1.1.1], the boundedness of $\mathcal{A}^{\infty}-\tilde{\mathcal{A}}^{\infty}$ and the dominated convergence theorem, the above relation holds for $f(x)=\mathbb{1}_{O}(x)$ for any open set $O \subseteq \mathbb{R}^{d}$. Thus, it also holds for any $f \in B_{b}\left(\mathbb{R}^{d}\right)$. Recall also that $P_{t} f \in C_{b}\left(\mathbb{R}^{d}\right)$ for every $f \in C_{b}\left(\mathbb{R}^{d}\right)$ and every $t \geq 0$ (see [69, Corollary 3.4]). Now, according to (i), there is a measurable function $C_{\varepsilon}:(0, \infty) \rightarrow(0, \infty)$ such that $\int_{0}^{t} C_{\varepsilon}(s) \mathrm{d} s<\infty$ and $\left\|\tilde{P}_{t} f\right\|_{\varepsilon} \leq C_{\varepsilon}(t)\|f\|_{\infty}$ for all $t>0$ and all $\tau$-periodic $f \in C_{b}\left(\mathbb{R}^{d}\right)$. Thus, for fixed $\tau$-periodic $f \in C_{b}\left(\mathbb{R}^{d}\right), P_{t} f \in C_{b}^{\varepsilon}\left(\mathbb{R}^{d}\right)$ and 


$$
\begin{aligned}
& \left\|P_{t} f\right\|_{\varepsilon} \leq\left\|\tilde{P}_{t} f\right\|_{\varepsilon}+\int_{0}^{t}\left\|\tilde{P}_{s}\left(\mathcal{A}^{\infty}-\tilde{\mathcal{A}}^{\infty}\right) P_{t-s} f\right\|_{\varepsilon} \mathrm{d} s \\
& \quad \leq \bar{C}_{\varepsilon}(t)\|f\|_{\infty},
\end{aligned}
$$

where $\bar{C}_{\varepsilon}(t)=C_{\varepsilon}(t)+\left\|\mathcal{A}^{\infty}-\tilde{\mathcal{A}}^{\infty}\right\| \int_{0}^{t} C_{\varepsilon}(s) \mathrm{d} s$. Also,

$$
\int_{0}^{t} \bar{C}(s) \mathrm{d} s \leq\left(1+t\left\|\mathcal{A}^{\infty}-\tilde{\mathcal{A}}^{\infty}\right\|\right) \int_{0}^{t} C(s) \mathrm{d} s, \quad t>0,
$$

where $\left\|\mathcal{A}^{\infty}-\tilde{\mathcal{A}}^{\infty}\right\|$ stands for the operator norm of $\mathcal{A}^{\infty}-\tilde{\mathcal{A}}^{\infty}$. Thus, $\left\{X_{t}\right\}_{t \geq 0}$ satisfies (C4)(i) with $\psi(r)=r^{\varepsilon}$. Therefore, if additionally $\int_{B_{1}^{c}(0)} y v(\cdot, \mathrm{d} y) \in$ $C_{b}^{\varepsilon}\left(\mathbb{R}^{d}\right)$, the conclusion of Theorem 1.4 holds true.

(iii) (Pure-jump LTPs) In the pure jump case, sufficient conditions for (C4)(i) are given in [53, Theorem 1.1]. Also, when the underlying process is given as a solution to an SDE of the form Eq. (1.7), we refer to [51,52,54] and the references therein.

To construct an example satisfying (C4)(ii), we can again employ a perturbation method. Let $\left\{X_{t}\right\}_{t \geq 0}$ and $\left\{\tilde{X}_{t}\right\}_{t \geq 0}$ be LTPs with semigroups $\left\{P_{t}\right\}_{t \geq 0}$ and $\left\{\tilde{P}_{t}\right\}_{t \geq 0}$, and $B_{b}$-generators $\left(\mathcal{A}^{b}, \mathcal{D}_{\mathcal{A}^{b}}\right)$ and $\left(\tilde{\mathcal{A}}^{b}, \mathcal{D}_{\tilde{\mathcal{A}}^{b}}\right)$, respectively. Assume that $\mathcal{A}^{b}$ satisfies (C4)(ii) for some Hölder exponents $\psi(r)$ and $\varphi(r)$. Further, assume that $\mathcal{A}^{b}-\tilde{\mathcal{A}}^{b}$ is a bounded operator on $\left(B_{b}\left(\mathbb{R}^{d}\right),\|\cdot\|_{\infty}\right)$, and that $\left(\mathcal{A}^{b}-\tilde{\mathcal{A}}^{b}\right) f \in C_{b}\left(\mathbb{R}^{d}\right)$ for every $f \in C_{b}\left(\mathbb{R}^{d}\right)$. Then,

$$
\tilde{P}_{t} f=P_{t} f+\int_{0}^{t} P_{s}\left(\mathcal{A}^{b}-\tilde{\mathcal{A}}^{b}\right) \tilde{P}_{t-s} f \mathrm{~d} s, \quad f \in \mathcal{D}_{\mathcal{A}^{b}} \cap \mathcal{D}_{\tilde{\mathcal{A}}^{b}} .
$$

Similarly as before, the above relation holds for all $f \in B_{b}\left(\mathbb{R}^{d}\right)$. Thus, for any $\lambda>0$ and any $\tau$-periodic $f \in C_{b}\left(\mathbb{R}^{d}\right)$,

$$
\tilde{R}_{\lambda}^{\tau} f_{\tau}=R_{\lambda}^{\tau} f_{\tau}+R_{\lambda}^{\tau}\left(\mathcal{A}^{b}-\tilde{\mathcal{A}}^{b}\right) \tilde{R}_{\lambda}^{\tau} f_{\tau}
$$

Assume now that $\left\{\tilde{P}_{t}\right\}_{t \geq 0}$ satisfies (C4)(i) with $\psi(r)$, and that $\left(\mathcal{A}^{b}-\tilde{\mathcal{A}}^{b}\right) f \in$ $C_{b}^{\psi}\left(\mathbb{R}^{d}\right)$ for every $f \in C_{b}^{\psi}\left(\mathbb{R}^{d}\right)$. Then, according to the proof of Theorem 1.4 (a), for any $\tau$-periodic $f \in C_{b}\left(\mathbb{R}^{d}\right)$ with $\int_{\mathbb{T}_{\tau}^{d}} f_{\tau}(x) \pi(\mathrm{d} x)=0, \tilde{R}_{\lambda}^{\tau} f \in C^{\psi}\left(\mathbb{T}_{\tau}^{d}\right)$ and so $\left(\mathcal{A}^{b}-\tilde{\mathcal{A}}^{b}\right) \tilde{R}_{\lambda}^{\tau} f_{\tau} \in C^{\psi}\left(\mathbb{T}_{\tau}^{d}\right)$. Hence, for any $\tau$-periodic $f \in C_{b}^{\psi}\left(\mathbb{R}^{d}\right)$, $\tilde{R}_{\lambda}^{\tau} f_{\tau} \in C^{\varphi \psi}\left(\mathbb{T}_{\tau}^{d}\right)$, that is, the corresponding $\tau$-periodic extension is a solution to Eq. (1.11). Finally, uniqueness follows from the fact that any solution $u(x)$ to Eq. (1.11) must have the representation $\int_{0}^{\infty} e^{-\lambda t} \tilde{P}_{t} f \mathrm{~d} t$, since $u=\left(\lambda-\tilde{\mathcal{A}}^{b}\right)^{-1} f$.

Below we give concrete examples of LTPs $\left\{X_{t}\right\}_{t \geq 0}$ and $\left\{\tilde{X}_{t}\right\}_{t \geq 0}$ satisfying the above assumptions. Let $\varphi:(0, \infty) \rightarrow(0, \infty)$ be increasing, and such that $\varphi(1)=1$ and

(a) there are $0<\underline{\alpha} \leq \bar{\alpha}<1, \underline{\kappa} \in(0,1]$ and $\bar{\kappa} \in[1, \infty)$, such that

$$
\underline{\kappa} \lambda^{2 \underline{\alpha}} \varphi(r) \leq \varphi(\lambda r) \leq \bar{\kappa} \lambda^{2 \bar{\alpha}} \varphi(r), \quad \lambda \geq 1, r \in(0,1]
$$


(b) $\int_{1}^{\infty} \frac{1}{r \varphi(r)} \mathrm{d} r<\infty$.

Then, by (a), $\lim _{r \rightarrow 0} \varphi(r)=0$, and so $\varphi(r)$ is a Hölder exponent with $\left[m_{\varphi}, M_{\varphi}\right]$ $\subseteq[2 \underline{\alpha}, 2 \bar{\alpha}] \subset(0,2)$. Further, let $n: \mathbb{R}^{d} \backslash\{0\} \rightarrow[\underline{\Gamma}, \bar{\Gamma}]$, with $0 \leq \underline{\Gamma} \leq \bar{\Gamma}<\infty$, be measurable. Then, thanks to (a) and (b),

$$
v_{0}(\mathrm{~d} y):=\frac{n(y)}{\varphi(|y|)|y|^{d}} \mathrm{~d} y
$$

is a Lévy measure. Denote the Lévy process generated by the Lévy triplet $\left(0,0, v_{0}(\mathrm{~d} y)\right)$ by $\left\{X_{t}\right\}_{t \geq 0}$. Also, let $\left(\mathcal{A}^{b}, \mathcal{D}_{\mathcal{A}^{b}}\right)$ be the corresponding $B_{b}$-generator. Then, $\left\{X_{t}\right\}_{t \geq 0}$ satisfies $(\mathbf{C 4})($ ii) for any Hölder exponent $\psi(r)$ such that $\left[m_{\psi}, M_{\psi}\right] \subset(0,1)$ and $\left[m_{\varphi \psi}, M_{\varphi \psi}\right] \cap \mathbb{N}=\emptyset$. Namely, since $\left\{X_{t}\right\}_{t \geq 0}$ has $\tau$-periodic (actually constant) coefficients, the corresponding projection (with respect to $\left.\Pi_{\tau}(x)\right)$ on $\mathbb{T}_{\tau}^{d}$ is again a strong Markov process. Moreover, according to [72, Proposition 2.2] (see also [48, Theorem 2.1]) and Proposition 3.1, it is also strong Feller and open-set irreducible so it satisfies Eq. (1.8). Now, for any $\lambda>0$ and any $\tau$-periodic $f \in B_{b}\left(\mathbb{R}^{d}\right)$, we see as before that the $\tau$-periodic extension $u_{\lambda, f}(x)$ of $R_{\lambda}^{\tau} f_{\tau}(x)$ solves $\lambda u_{\lambda, f}-\mathcal{A}^{b} u_{\lambda, f}=f$. If $f \in C_{b}^{\psi}\left(\mathbb{R}^{d}\right)$ for some Hölder exponent $\psi(r)$ such that $\left[m_{\psi}, M_{\psi}\right] \subset(0,1)$, then, according to [72, Proposition 2.2] and the proof of [6, Propositions 3.5 and 3.6], $u_{\lambda, f} \in C_{b}^{\varphi \psi}\left(\mathbb{R}^{d}\right)$ provided $\left[m_{\varphi \psi}, M_{\varphi \psi}\right] \cap \mathbb{N}=\emptyset$. Let us remark that in the proofs of [6, Propositions 3.5 and 3.6] the authors require the scaling property Eq. (3.3) of $\varphi(r)$ for all $r \in(0, \infty)$, and the additional assumptions that $n(y) \equiv c$ for some $c>0$ and that $\phi(r)=\varphi\left(r^{-1 / 2}\right)^{-1}$ is a Bernstein function, that is, $(-1)^{n} \phi^{(n)}(r) \leq 0$ for every $n \in \mathbb{N}_{0}$. They essentially use this property in order to apply [6, Corollary 3.2] via the regularity of semigroups associated with subordinated Brownian motions. However, the statement of this corollary has been proved in [72, Proposition 2.2] under the scaling condition in Eq. (3.3). Finally, uniqueness follows from (a straightforward adaptation of) [63, Proposition 3.2] (by taking $b(x) \equiv 0$ ). A typical example of the function $\varphi(r)$ satisfying the above assumptions is given by $\varphi(r)=r^{\alpha} \log ^{\beta}\left(\mathrm{e}-1+r^{-1}\right)$ with $\alpha \in(0,2)$ and $\beta \in \mathbb{R}$. According to [72, Proposition 2.2], there is $c>0$ such that for all $t \in(0,1]$ and $f \in B_{b}\left(\mathbb{R}^{d}\right),\left\|\nabla P_{t} f\right\|_{\infty} \leq c\left(\varphi^{-1}(t)\right)^{-1}$. Therefore, we have that (1) if $\alpha \in(1,2)$ or $\alpha=1$ and $\beta<-\alpha$, then (C4)(i) is satisfied and Theorem 1.4 (b)(2) holds with $\psi(r)=r^{\theta_{1}} \log ^{\theta_{2}}\left(1+r^{-1}\right)$ for any $\theta_{1} \in(0,1)$ and $\theta_{2} \in \mathbb{R}$; (2) if $\alpha \in(0,1)$, then (C4)(i) is satisfied with $\psi(r)=r^{\theta_{1}} \log ^{\theta_{2}}\left(1+r^{-1}\right)$ for any $\theta_{1} \in(0, \alpha)$ and $\theta_{2} \in \mathbb{R}$, and Theorem 1.4 (b)(3) holds.

Also, as we have commented above, $\left\{X_{t}\right\}_{t \geq 0}$ satisfies (C4)(ii) if $\theta_{1}$ is such that $\alpha+\theta_{1} \notin \mathbb{N}$.

Further, let $\left\{\tilde{X}_{t}\right\}_{t \geq 0}$ be a LTP generated by $(0,0, v(x, \mathrm{~d} y))$ with

$$
\nu(x, \mathrm{~d} y)=\mathbb{1}_{B_{1}(0)}(y) \nu_{0}(\mathrm{~d} y)+\frac{\gamma(x, y)}{\tilde{\varphi}(|y|)|y|^{d}} \mathbb{1}_{B_{1}^{c}(0)}(y) \mathrm{d} y,
$$


where $\tilde{\varphi}:[1, \infty) \rightarrow(0, \infty)$ satisfies that $\int_{1}^{\infty} \frac{1}{r \tilde{\varphi}(r)} \mathrm{d} r<\infty$, and $\gamma(x, y)$ is non-negative, bounded and such that $x \mapsto \gamma(x, y)$ is continuous for almost every $y \in \mathbb{R}^{d}$ on $B_{1}^{c}(0)$ (see (iv) in the discussion on the strong Feller property above that this Lévy kernel generates a LTP). Denote by $\left(\tilde{\mathcal{A}}^{b}, \mathcal{D}_{\tilde{\mathcal{A}}^{b}}\right)$ the corresponding $B_{b}$-generator. It is easy to see that $\mathcal{A}^{b}-\tilde{\mathcal{A}}^{b}$ is bounded on $\left(B_{b}\left(\mathbb{R}^{d}\right),\|\cdot\|_{\infty}\right)$, and $\left(\mathcal{A}^{b}-\tilde{\mathcal{A}}^{b}\right) f \in C_{b}\left(\mathbb{R}^{d}\right)$ for every $f \in C_{b}\left(\mathbb{R}^{d}\right)$. Furthermore, $\left(\mathcal{A}^{b}-\tilde{\mathcal{A}}^{b}\right) f \in C_{b}^{\psi}\left(\mathbb{R}^{d}\right)$ for every $f \in C_{b}^{\psi}\left(\mathbb{R}^{d}\right)$ if we additionally assume that for almost all $y \in \mathbb{R}^{d}$ on $B_{1}^{c}(0), x \mapsto \gamma(x, y)$ is of class $C_{b}^{\psi}\left(\mathbb{R}^{d}\right)$. With these at hand, we can follow the argument in (ii) to check that (C4) is satisfied, and so the conclusion of Theorem 1.4 holds.

\section{Acknowledgements}

Open Access funding provided by Projekt DEAL. Financial support through the Alexander-von-Humboldt Foundation (No. HRV 1151902 HFST-E) and Croatian Science Foundation under project 8958 (for N. Sandric), Croatian Science Foundation under project 8958 (for I. Valentic), and the National Natural Science Foundation of China (No. 11831014), the Program for Probability and Statistics: Theory and Application (No. IRTL1704) and the Program for Innovative Research Team in Science and Technology in Fujian Province University (IRTSTFJ) (for J. Wang) are gratefully acknowledged. We also thank the anonymous referees for the helpful comments that have led to significant improvements of the results in the article.

Open Access. This article is licensed under a Creative Commons Attribution 4.0 International License, which permits use, sharing, adaptation, distribution and reproduction in any medium or format, as long as you give appropriate credit to the original author(s) and the source, provide a link to the Creative Commons licence, and indicate if changes were made. The images or other third party material in this article are included in the article's Creative Commons licence, unless indicated otherwise in a credit line to the material. If material is not included in the article's Creative Commons licence and your intended use is not permitted by statutory regulation or exceeds the permitted use, you will need to obtain permission directly from the copyright holder. To view a copy of this licence, visit http://creativecommons.org/licenses/ by/4.0/.

Publisher's Note Springer Nature remains neutral with regard to jurisdictional claims in published maps and institutional affiliations.

\section{REFERENCES}

[1] A. Arapostathis, G. Pang, and N. Sandrić. Ergodicity of a Lévy-driven SDE arising from multiclass many-server queues, Ann. Appl. Probab. 29 (2019), no. 2, 1070-1126.

[2] M. Arisawa, Quasi-periodic homogenizations for second-order Hamilton-Jacobi-Bellman equations, Adv. Math. Sci. Appl. 11 (2001), no. 1, 465-480.

[3] M. Arisawa, Homogenization of a class of integro-differential equations with Lévy operators, Comm. Partial Differential Equations 34 (2009), no. 7-9, 617-624.

[4] M. Arisawa, Homogenizations of integro-differential equations with Lévy operators with asymmetric and degenerate densities, Proc. Roy. Soc. Edinburgh Sect. A 142 (2012), no. 5, 917-943. 
[5] D. G. Aronson, Bounds for the fundamental solution of a parabolic equation, Bull. Amer. Math. Soc. 73 (1967), no. 6, 890-896.

[6] J. Bae and M. Kassmann, Schauder estimates in generalized Hölder spaces, ArXiv e-prints $\mathbf{1 5 0 5 . 0 5 4 9 8}$ (2015).

[7] G. Barles, E. Chasseigne, A. Ciomaga, and C. Imbert, Large time behavior of periodic viscosity solutions for uniformly parabolic integro-differential equations, Calc. Var. Partial Differential Equations 50 (2014), no. 1-2, 283-304.

[8] M. T. Barlow, R. F. Bass, Z. Q. Chen, and M. Kassmann, Non-local Dirichlet forms and symmetric jump processes, Trans. Amer. Math. Soc. 361 (2009), no. 4, 1963-1999.

[9] M. T. Barlow, A. Grigor'yan, and T. Kumagai, Heat kernel upper bounds for jump processes and the first exit time, J. Reine Angew. Math. 626 (2009), 135-157.

[10] A. Bensoussan, J L. Lions, and G. C. Papanicolaou, Asymptotic Analysis for Periodic Structures, North-Holland Publishing Co., Amsterdam, 1978.

[11] R. N. Bhattacharya, A central limit theorem for diffusions with periodic coefficients, Ann. Probab. 13 (1985), no. 2, 385-396.

[12] N. H. Bingham, C. M. Goldie, and J. L. Teugels, Regular variation, Encyclopedia of Mathematics and its Applications, vol. 27, Cambridge University Press, Cambridge, 1987.

[13] R. M. Blumenthal and R. K. Getoor, Markov Processes and Potential Theory, Academic Press, New York-London, 1968.

[14] B. Böttcher, R. L. Schilling, and J. Wang, Lévy Matters. III. , Springer, Cham, 2013.

[15] L. Bălilescu, A. Ghosh, and T. Ghosh, Homogenization for non-local elliptic operators in both perforated and non-perforated domains, Zeitschrift für angewandte Mathematik und Physik 70 (2019), Article number: 171.

[16] G. A. Chechkin, A. L. Piatnitski, and A. S. Shamaev, Homogenization, American Mathematical Society, Providence, RI, 2007.

[17] X. Chen, Z. Q. Chen, and J. Wang, Heat kernel for non-local operators with variable order, Stochastic Process. Appl 130 (2020), no. 6, 3574-3647.

[18] Z. Q. Chen, E. Hu, L. Xie, and X. Zhang, Heat kernels for non-symmetric diffusion operators with jumps, J. Differential Equations 263 (2017), no. 10, 6576-6634.

[19] Z. Q. Chen and X. Zhang, Heat kernels and analyticity of non-symmetric jump diffusion semigroups, Probab. Theory Related Fields 165 (2016), no. 1-2, 267-312.

[20] Z. Q. Chen and X. Zhang, Heat kernels for time-dependent non-symmetric stable-like operators, J. Math. Anal. Appl. 465 (2018), no. 1, 1-21.

[21] K. L. Chung and J. B. Walsh, Markov Processes, Brownian Motion, and Time Symmetry, second ed., Springer, New York, 2005.

[22] E. Çinlar and J. Jacod, Representation of semimartingale Markov processes in terms of Wiener processes and Poisson random measures, Seminar on Stochastic Processes, Birkhäuser, Boston, Mass., 1981, pp. 159-242.

[23] D. Cioranescu and P. Donato, An Introduction to Homogenization, The Clarendon Press, Oxford University Press, New York, 1999.

[24] Ph. Courrége, Sur la forme intégro-différentielle des opérateurs de $C_{K}^{\infty}$ dans $C$ satisfaisant au principe du maximum, Sém. Théorie du Potentiel exposé 2 (1965-1966), 38 pp.

[25] J. L. Doob, Stochastic Processes, John Wiley \& Sons, Inc., New York; Chapman \& Hall, Limited, London, 1953.

[26] R. Durrett, Stochastic Calculus, CRC Press, Boca Raton, FL, 1996.

[27] M. Errami, F. Russo, and P. Vallois, Itô's formula for $C^{1, \lambda}$-functions of a càdlàg process and related calculus, Probab. Theory Related Fields 122 (2002), no. 2, 191-221.

[28] S. N. Ethier and T. G. Kurtz, Markov Processes, John Wiley \& Sons Inc., New York, 1986.

[29] J. Fernández Bonder, A. Ritorto, and A. M. Salort, H-convergence result for nonlocal elliptic-type problems via Tartar's method, SIAM J. Math. Anal. 49 (2017), no. 4, 2387-2408.

[30] M. Focardi, Aperiodic fractional obstacle problems, Adv. Math. 225 (2010), no. 6, 3502-3544.

[31] B. Franke, The scaling limit behaviour of periodic stable-like processes, Bernoulli 12 (2006), no. 3, 551-570.

[32] B. Franke, Correction to: "The scaling limit behaviour of periodic stable-like processes" [Bernoulli 12 (2006), no. 3, 551-570], Bernoulli 13 (2007), no. 2, 600. 
[33] B. Franke, A functional non-central limit theorem for jump-diffusions with periodic coefficients driven by stable Lévy-noise, J. Theoret. Probab. 20 (2007), no. 4, 1087-1100.

[34] A. Friedman, Stochastic Differential Equations and Applications. Vol. 1, Academic Press [Harcourt Brace Jovanovich, Publishers], New York-London, 1975.

[35] T. Fujiwara and M. Tomisaki, Martingale approach to limit theorems for jump processes, Stochastics Stochastics Rep. 50 (1994), no. 1-2, 35-64.

[36] T. Grzywny and K. Szczypkowski, Heat kernels of non-symmetric Lévy-type operators, J. Differential Equations 267 (2019), no. 10, 6004-6064.

[37] M. Horie, T. Inuzuka, and H. Tanaka, Homogenization of certain one-dimensional discontinuous Markov processes, Hiroshima Math. J. 7 (1977), no. 2, 629-641.

[38] Q. Huang, J. Duan, and R. Song, Homogenization of nonlocal partial differential equations related to stochastic differential equations with Lévy noise, ArXiv e-prints $\mathbf{1 8 0 4 . 0 6 5 5 5}$ (2018).

[39] Q. Huang, J. Duan, and R. Song, Homogenization of stable-like Feller processes, ArXiv e-prints $\mathbf{1 8 1 2 . 1 1 6 2 4}$ (2018).

[40] N. Ikeda and S. Watanabe, Stochastic Differential Equations and Diffusion Processes, second ed., North-Holland Publishing Co., Amsterdam; Kodansha, Ltd., Tokyo, 1989.

[41] N. Jacob, Pseudo Differential Operators and Markov Processes. Vol. I, Imperial College Press, London, 2001.

[42] N. Jacob, Pseudo Differential Operators and Markov Processes. Vol. III, Imperial College Press, London, 2005.

[43] J. Jacod and A. N. Shiryaev, Limit Theorems for Stochastic Processes, second ed., vol. 288, SpringerVerlag, Berlin, 2003.

[44] V. V. Jikov, S. M. Kozlov, and O. A. Oleŭnik, Homogenization of Differential Operators and Integral Functionals, Springer-Verlag, Berlin, 1994.

[45] M. Kassmann, A. Piatnitski, and E. Zhizhina, Homogenization of Lévy-type operators with oscillating coefficients, SIAM J. Math. Anal. 51 (2019), no. 5, 3641-3665.

[46] P. Kim and J. Lee, Heat kernels of non-symmetric jump processes with exponentially decaying jumping kernel, Stochastic Process. Appl. 129 (2019), no. 6, 2130-2173.

[47] P. Kim, R. Song, and Z. Vondraček, Heat kernels of non-symmetric jump processes: beyond the stable case, Potential Anal. 49 (2018), no. 1, 37-90.

[48] V. Knopova and R. L. Schilling, A note on the existence of transition probability densities of Lévy processes, Forum Math. 25 (2013), no. 1, 125-149.

[49] V. N. Kolokoltsov, Markov Processes, Semigroups and Generators, vol. 38, Walter de Gruyter \& Co., Berlin, 2011.

[50] Y. Kwon and C. Lee, Strong Feller property and irreducibility of diffusions with jumps, Stochastics Stochastics Rep. 67 (1999), no. 1-2, 147-157.

[51] M. Liang, R. L. Schilling, and J. Wang, A unified approach to coupling SDEs driven by Lévy noise and some applications, Bernoulli 26 (2020), no. 1, 664-693.

[52] M. Liang and J. Wang, Gradient estimates and ergodicity for SDEs driven by multiplicative Lévy noises via coupling, Stochastic Process. Appl. 130 (2020), no. 5, 3053-3094.

[53] M. Liang and J. Wang, Spatial regularity of semigroups generated by Lévy type operators, Math. Nachr. 292 (2019), no. 7, 1551-1566.

[54] D. Luo and J. Wang, Refined basic couplings and Wasserstein-type distances for SDEs with Lévy noises, Stochastic Process. Appl. 129 (2019), no. 9, 3129-3173.

[55] H. Masuda, Ergodicity and exponential $\beta$-mixing bounds for multidimensional diffusions with jumps, Stochastic Process. Appl. 117 (2007), no. 1, 35-56.

[56] H. Masuda, Erratum to: "Ergodicity and exponential $\beta$-mixing bound for multidimensional diffusions with jumps” [Stochastic Process. Appl. 117 (2007) 35-56], Stochastic Process. Appl. 119 (2009), no. 2, 676-678.

[57] S. P. Meyn and R. L. Tweedie, Stability of Markovian Mrocesses. II. Continuous-time processes and sampled chains, Adv. Appl. Probab. 25 (1993), no. 3, 487-517.

[58] S. P. Meyn and R. L. Tweedie, Stability of Markovian processes. III. Foster-Lyapunov criteria for continuous-time processes, Adv. Appl. Probab. 25 (1993), no. 3, 518-548.

[59] J. Nash, Continuity of solutions of parabolic and elliptic equations, Amer. J. Math. 80 (1958), no. 4, 931-954. 
[60] Y. Ogura, M. Tomisaki, and M. Tsuchiya, Existence of a strong solution for an integro-differential equation and superposition of diffusion processes, Stochastics in finite and infinite dimensions, Trends Math., Birkhäuser Boston, Boston, MA, 2001, pp. 341-359.

[61] G. Pang and N. Sandrić, Ergodicity and fluctuations of a fluid particle driven by diffusions with jumps, Commun. Math. Sci. 14 (2016), no. 2, 327-362.

[62] A. Piatnitski and E. Zhizhina, Periodic homogenization of nonlocal operators with a convolutiontype kernel, SIAM J. Math. Anal. 49 (2017), no. 1, 64-81.

[63] E. Priola, Pathwise uniqueness for singular SDEs driven by stable processes, Osaka J. Math. 49 (2012), no. 2, 421-447.

[64] R. Rhodes and V. Vargas, Scaling limits for symmetric Itô-Lévy processes in random medium, Stochastic Process. Appl. 119 (2009), no. 12, 4004-4033.

[65] L. C. G. Rogers and D. Williams, Diffusions, Markov Processes, and Martingales. Vol. 1, Cambridge University Press, Cambridge, 2000.

[66] L. C. G. Rogers and D. Williams, Diffusions, Markov Processes, and Martingales. Vol. 2, Cambridge University Press, Cambridge, 2000.

[67] N. Sandrić, Homogenization of periodic diffusion with small jumps, J. Math. Anal. Appl. 435 (2016), no. 1, 551-577.

[68] K. Sato, Lévy Processes and Infinitely Divisible Distributions, Cambridge University Press, Cambridge, 1999.

[69] R. L. Schilling, Conservativeness and extensions of Feller semigroups, Positivity 2 (1998), 239-256.

[70] R. L. Schilling, Growth and Hölder conditions for the sample paths of Feller processes, Probab. Theory Related Fields 112 (1998), no. 4, 565-611.

[71] R. L. Schilling and A. Schnurr, The symbol associated with the solution of a stochastic differential equation, Electron. J. Probab. 15 (2010), 1369-1393.

[72] R. L. Schilling, P. Sztonyk, and J. Wang, Coupling property and gradient estimates of Lévy processes via the symbol, Bernoulli 18 (2012), no. 4, 1128-1149.

[73] R. L. Schilling and T. Uemura, Homogenization of symmetric Lévy processes on $\mathbb{R}^{d}$, to appear in Revue Roumaine de Mathématiques Pures et Appliquées, ArXiv e-prints 1808.01667 (2018).

[74] R. L. Schilling and J. Wang, Strong Feller continuity of Feller processes and semigroups, Infin. Dimens. Anal. Quantum Probab. Relat. Top. 15 (2012), no. 2, 1250010, 28.

[75] R. W. Schwab, Periodic homogenization for nonlinear integro-differential equations, SIAM J. Math. Anal. 42 (2010), no. 6, 2652-2680.

[76] R. W. Schwab, Stochastic homogenization for some nonlinear integro-differential equations, Comm. Partial Differential Equations 38 (2013), no. 2, 171-198.

[77] Y. Shiozawa and T. Uemura, Stability of the Feller property for non-local operators under bounded perturbations, Glas. Mat. Ser. III 45 (2010), no. 1, 155-172.

[78] T. Simon, Support theorem for jump processes, Stochastic Process. Appl. 89 (2000), no. 1, 1-30.

[79] D. W. Stroock, Diffusion processes associated with Lévy generators, Z. Wahrscheinlichkeitstheorie und Verw. Gebiete 32 (1975), no. 3, 209-244.

[80] D. W. Stroock, Diffusion semigroups corresponding to uniformly elliptic divergence form operators, Séminaire de Probabilités, XXII, Lecture Notes in Math., vol. 1321, Springer, Berlin, 1988, pp. 316347.

[81] L. C. Tartar, The General Theory of Homogenization, Springer-Verlag, Berlin; UMI, Bologna, 2009.

[82] M. Tomisaki, Homogenization of càdlàg processes, J. Math. Soc. Japan 44 (1992), no. 2, 281-305.

[83] R. L. Tweedie, Topological conditions enabling use of Harris methods in discrete and continuous time, Acta Appl. Math. 34 (1994), no. 1-2, 175-188.

[84] L. Wang and X. Zhang. Harnack inequalities for SDEs driven by cylindrical $\alpha$-stable processes, Potential Anal 42 (2015), 657-669, 2015.

[85] H. Watanabe, Potential operator of a recurrent strong Feller process in the strict sense and boundary value problem, J. Math. Soc. Japan 16 (1964), no. 2, 83-95. 
Nikola Sandrić

Institut für Mathematische Stochastik

Technische Universität Dresden

Dresden

Germany

E-mail: nsandric@math.hr

Nikola Sandrić and Ivana Valentić

Deparmtent of Mathematics

University of Zagreb

Zagreb

Croatia

E-mail: ivana.valentic@math.hr

Jian Wang

College of Mathematics and Informatics, Fujian Key Laboratory of Mathematical Analysis and Applications (FJKLMAA), Center for Applied Mathematics of Fujian Province (FJNU)

Fujian Normal University

Fuzhou

People's Republic of China

E-mail: jianwang@fjnu.edu.cn 